Canadian University Music Review

Revue de musique des universités canadiennes

\title{
L'adaptation d'une forme à un style : les thèmes à retour dans la musique instrumentale de Brahms
}

\section{François de Médicis}

Volume 20, numéro 2, 2000

URI : https://id.erudit.org/iderudit/1014458ar

DOI : https://doi.org/10.7202/1014458ar

Aller au sommaire du numéro

Éditeur(s)

Canadian University Music Society / Société de musique des universités canadiennes

ISSN

0710-0353 (imprimé)

2291-2436 (numérique)

Découvrir la revue

Citer cet article

de Médicis, F. (2000). L'adaptation d'une forme à un style : les thèmes à retour dans la musique instrumentale de Brahms. Canadian University Music Review / Revue de musique des universités canadiennes, 20(2), 45-79.

https://doi.org/10.7202/1014458ar
Résumé de l'article

La difficulté de situer Brahms dans le camp des conservateurs ou des progressistes résulte probablement du caractère protéiforme de sa musique. Cette flexibilité caractérise également les structures à retour, qui sont les formes thématiques les plus abondantes dans la musique instrumentale de Brahms. Ces formes sont fondées sur le retour d'une idée initiale après un matériau intermédiaire, comme dans la période ou la forme ternaire. L’analyse des deux paramètres fondamentaux de ces structures, le retour et la cadence, montre que leur faveur peut s'expliquer par une bonne adaptation aux nouvelles exigences stylistiques du romantisme, comme le rejet de conventions trop prévisibles du discours, et l'élargissement de l'espace tonal.
All Rights Reserved (C Canadian University Music Society / Société de musique des universités canadiennes, 2000
Ce document est protégé par la loi sur le droit d'auteur. L'utilisation des services d'Érudit (y compris la reproduction) est assujettie à sa politique d'utilisation que vous pouvez consulter en ligne.

https://apropos.erudit.org/fr/usagers/politique-dutilisation/ 


\title{
L'ADAPTATION D'UNE FORME À UN STYLE : LES THÈMES À RETOUR DANS LA MUSIQUE INSTRUMENTALE DE BRAHMS
}

\author{
François de Médicis
}

\section{INTRODUCTION}

Il semble que la contribution de Johannes Brahms à l'histoire de la musique soit difficile à définir, et prête souvent même à des malentendus. En 1853, Schumann saluait en lui le messie musical qui allait poursuivre la voie ouverte par Beethoven ${ }^{1}$; un échange houleux dans la presse des années 1860 opposait le compositeur au clan de Wagner et Liszt, qui le taxaient de conservatisme ${ }^{2}$; en 1933, Schönberg avançait une vision réconciliatrice en le considérant comme un progressiste au même titre que Wagner ${ }^{3}$; et, plus récemment, Reinhold Brinkmann faisait valoir la dimension crépusculaire, mélancolique de son art de romantique attardé ${ }^{4}$. On peut probablement imputer une telle ambiguitté, du moins en partie, au caractère protéiforme de la musique de Brahms, dans laquelle cohabitent une grande variété de structures. Un bon exemple de ce trait s'observe dans sa musique instrumentale par l'utilisation d'un type formel qu'on appellera ici «structure thématique à retour ». Cette catégorie est fondée sur le principe du retour de l'idée initiale d'un thème et comprend deux structures principales : (1) la période, dont l'idée de base présentée au début de l'antécédent fait l'objet d'un retour au début du conséquent; et (2) la petite forme ternaire (ou $\mathrm{ABA}$ '), dont l'idée de base exposée au début de la section $\mathrm{A}$ est reprise au début de la section $\mathrm{A}^{\prime}$. Pour préserver l'homogénéité du corpus et profiter d'un cadre formel général bien connu, on s'intéressera aux structures à retour dans les œuvres instrumentales écrites dans la tradition de la sonate entendue au sens large, soit les cycles de mouvements de caractère sérieux, qui comprennent les sonates pour piano, la musique de chambre, les concertos, les sérénades et les symphonies. On laissera donc de côté les œuvres vocales et,

\footnotetext{
${ }^{1}$ Voir l'article « Neue Bahnen » (1853) de Robert Schumann, cité en traduction anglaise dans Robert Schumann on Music : A Selection from the Writings, traduit et édité par Henry Pleasants (New York : Dover Publications, 1988), 199-200.

${ }^{2}$ On peut lire un compte rendu de la controverse dans Alan Walker, Franz Liszt :Tome I (18111861) (Paris : Fayard, 1989), 814-46.

${ }^{3}$ Arnold Schönberg, «Brahms, le progressiste », dans Le style et l'idée (Paris : Buchet-Chastel, 1977), 305-43.

${ }^{4}$ Reinhold Brinkmann, Late Idyll : The Second Symphony of Johannes Brahms, traduit par Peter Palmer (Cambridge : Harvard University Press, 1995); version originale allemande dans MusikKonzept 70 (1990).
} 
parmi la musique instrumentale, les cycles de pièces brèves pour piano solo (op. 10,76, 79, 116-19) et piano à quatre mains (Valses, op. 39, 52 et 65; Danses hongroises sans numéro d'opus, etc.), les séries de variations (op. 9, 21, $\mathrm{n}^{\mathrm{os}} 1$ et 2 , et op. 23,24 , etc.) et les ouvertures pour orchestre (op. 80,81$)^{5}$.

Ce travail découle d'une observation : les structures à retour sont, de loin, les formes thématiques les plus abondantes dans la musique instrumentale de Brahms. Cette prédominance n'est pas donnée partout pour le répertoire instrumental, comme le montre un parallèle entre Brahms et les classiques. Les formes closes, parfois appelées formes vocales ( «song forms ») et représentées par des patrons formels comme $\mathrm{ABA}$ ou $\mathrm{AAB}$, sont rarement utilisées dans la forme sonate des classiques - surtout dans le premier mouvement des cycles instrumentaux. Leur utilisation est plus fréquente dans les autres mouvements, comme dans le thème d'un mouvement lent, le refrain ou le couplet d'un rondo (par exemple le thème initial du mouvement lent de la Sonate pour piano en fa majeur, op. $2, \mathrm{n}^{\circ} 1$, de Beethoven, ou le refrain du finale de son Quatuor à cordes en do mineur, op. 18, $\mathrm{n}^{\circ} 4$ ). Si d'autres structures à retour comme les périodes sont employées couramment dans les thèmes principal et subordonné de la forme sonate classique, elles adoptent généralement des proportions modestes, de 8 à 16 mesures, et sont occasionnellement allongées par processus additif lorsqu'un geste cadentiel est suivi d'une extension qui mène à une nouvelle tentative cadentielle qui, selon qu'elle est aboutie ou non, conclura le thème ou amènera une nouvelle extension ${ }^{6}$. On trouve un exemple de ce procédé chez Beethoven, dans le premier mouvement de la Sonate pour piano en mi bémol majeur, op. 7 (mes. 60-93).

Plusieurs auteurs ont déjà noté l'utilisation bien peu classique des formes closes dans les formes sonate de Brahms ${ }^{7}$. En fait, la présence de ces types dans la forme sonate se rattache à une prédilection plus générale des romantiques pour les structures à retour. De plus, les périodes de Brahms adoptent généralement des proportions plus imposantes que celles des classiques, et l'allongement résulte ici non pas d'un processus additif par extension comme chez ces derniers, mais d'une expansion interne de l'antécédent et du conséquent.

L'abondance et la variété des structures à retour chez Brahms soulèvent deux problèmes auxquels cet article tentera d'apporter une solution : d'abord, la nécessité de fournir un cadre général permettant de mettre un peu d'ordre dans la diversité d'organisation des structures à retour; puis le besoin de justifier la prédilection dont ce type formel fait l'objet. Pour traiter ces questions, le travail est divisé en trois parties. La première illustre la diversité des structures

\footnotetext{
${ }^{5}$ Pour une étude approfondie des structures à retour dans l'œuvre de Brahms et une bibliographie exhaustive du sujet, on consultera ma thèse de doctorat, «La spécificité des structures thématiques à retour dans l'œuvre instrumental de Brahms » (McGill University, 1997).

${ }^{6}$ Voir William Caplin, Classical Form : A Theory of Formal Functions for the Instrumental Music of Haydn, Mozart, and Beethoven (New York : Oxford University Press, 1998), 111.

7 Voir James Webster, « Schubert's Sonata Form and Brahms's First Maturity », NineteenthCentury Music 3 (1979) : 52-71; et Dahlhaus, « Issues in Composition », dans Between Romanticism and Modernism : Four Studies in the Music of Later Nineteenth Century, trad. par Mary Whittall (Berkeley et Los Angeles : University of California Press, 1980), 40-78. Webster explique ce trait dans les œuvres de la première maturité par l'influence de Schubert.
} 
à retour chez Brahms par l'analyse de trois thèmes, qui montre que si le compositeur écrit à l'occasion des structures similaires à celles des classiques, d'autres s'en distinguent radicalement. Les deuxième et troisième parties s'intéressent successivement au traitement du retour et à l'organisation cadentielle chez Brahms et les classiques. La comparaison tend à suggérer que la grande faveur des structures à retour dans la musique instrumentale de Brahms peut s'expliquer par une grande capacité d'adaptation aux nouvelles exigences stylistiques du romantisme, comme le rejet de conventions trop prévisibles et l'élargissement de l'espace tonal. De plus, cette discussion comporte d'importantes implications théoriques qui tendent à montrer le rôle primordial de l'organisation formelle dans la projection de la structure harmonique et tonale.

\section{LA DIVERSITÉ DE STRUCTURES À RETOUR CHEz BRAHMS}

Les deux principaux types de structures thématiques à retour - la période et la petite forme ternaire - ont souvent été décrites, et une des présentations les plus détaillées provient de la théorie des fonctions formelles de William Caplin. Cette approche, développée pour rendre compte de la musique classique, offre un bon point de départ pour amorcer une discussion de l'organisation formelle des thèmes de Brahms.

Selon la définition de Caplin, la période est constituée de deux phrases de quatre mesures distinctes (c'est-à-dire qui se succèdent sans élision) : un " antécédent " et un " conséquent ». Deux segments de deux mesures non identiques composent l'antécédent : le premier s'appelle «idée de base »; et le second, qui produit une cadence faible, s'appelle "idée contrastante ». Le conséquent reprend le matériau de l'antécédent avec quelques modifications de manière à se terminer par une cadence plus forte. La «cadence authentique parfaite », qui se termine avec la tonique à la basse et au soprano, offre le degré conclusif maximal. La « cadence authentique imparfaite » est plus faible, car l'aboutissement de la mélodie sur un degré différent de la tonique, généralement la tierce, est moins conclusive. La « demi-cadence » est la plus faible, car elle se termine sur une harmonie de dominante, qui est un degré moins stable que la tonique. Comme l'antécédent doit se terminer par une cadence plus faible que celle du conséquent, il peut utiliser une demi-cadence ou une cadence authentique imparfaite. Le conséquent se terminera le plus souvent par une cadence authentique parfaite, bien qu'une cadence authentique imparfaite soit aussi possible si l'antécédent se termine par une demi-cadence.

Un thème du dernier mouvement de la Symphonie $\mathrm{n}^{0} 1$ en do mineur, op. 68, de Brahms respecte d'assez près la structure de la période de huit mesures telle que décrite par Caplin (exemple 1). D'après cette théorie, le thème de la symphonie présente un antécédent et un conséquent de quatre mesures chacun (respectivement aux mes. 62-65 et 66-69), tous deux composés d'une idée de base et d'une idée contrastante de deux mesures. L'antécédent se termine par une demi-cadence (DC) à la mes. 65, soit une cadence plus faible que la cadence authentique parfaite (CAP) qui marque la fin du conséquent à la mes. 69 . 
ant. (4)

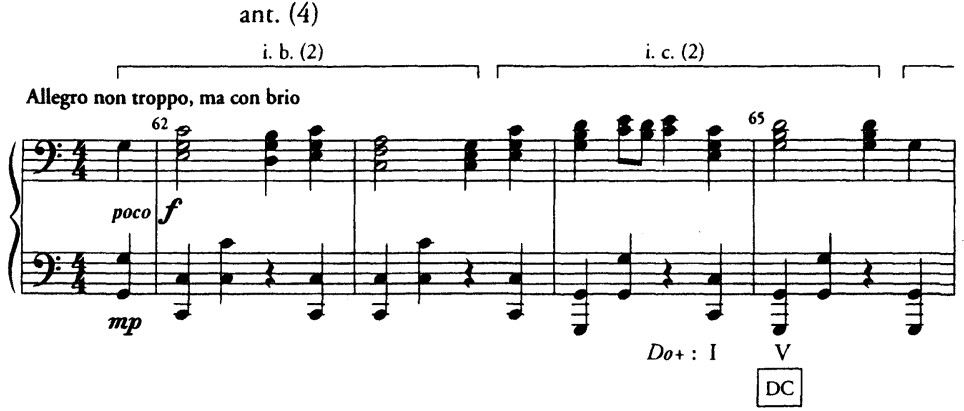

cons. (4)

i. b. (2) i. c. (2)

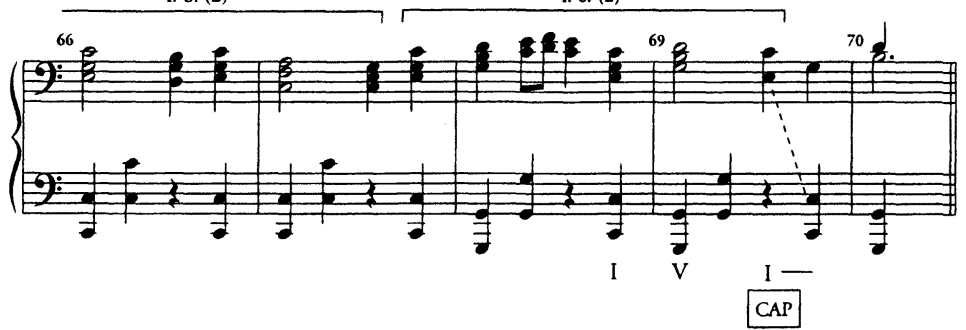

Exemple 1: Brahms, Symphonie $\mathrm{n}^{\circ} 1$ en do mineur, op. $68: 4^{\mathrm{e}}$ mouvement, mes. $62-70$

L'autre principal type de structure à retour - la petite forme ternaire - est bien illustré par le thème initial du mouvement lent du Concerto pour violon en ré majeur, op. 77 (exemple 2). Comme son nom l'indique, ce type formel est formé de trois parties distinctes qui présentent les fonctions " d'exposition » $(A)$, de « section médiane contrastante » $(B)$ et de « récapitulation » $\left(A^{\prime}\right)$. Le thème offre ici le découpage suivant: $\mathrm{A}=$ mes. $3-10, \mathrm{~B}=$ mes. $11-14, \mathrm{~A}^{\prime}=$ mes. 15-22. Selon le modèle classique, la section $A$ est généralement construite sur une forme thématique typique et se termine par une cadence authentique parfaite, soit dans le ton principal, soit dans un ton voisin ( $\mathrm{V}$ en majeur, III ou v en mineur). Dans la section médiane contrastante, l'absence de forme conventionnelle et l'insistance sur l'harmonie de dominante produisent un relâchement de la structure, une certaine instabilité par rapport à l'exposition. L'harmonie de dominante peut être soulignée de différentes manières : par une pédale sur cette harmonie, par une forme de prolongation appelée «station sur la dominante ${ }^{8}$, ou par une simple terminaison sur V (demi-cadence ou articulation non cadentielle, qualifiée « d'arrivée de la dominante »). Le relâchement structurel de la section $B$ est aussi fréquemment réalisé par l'emploi de séquences harmoniques. La récapitulation reprend le matériau mélodique et motivique de l'exposition, parfois de manière intégrale, souvent avec des modifications,

${ }^{8}$ Ce mot est l'équivalent français du Stehen auf der Dominante, forgé en allemand par Erwin Ratz, Einführung in die musikalische Formenlehre, $3^{e}$ éd. revue et augmentée (Vienne : Universal Edition, 1973), 25, et de sa traduction anglaise standing on dominant. 

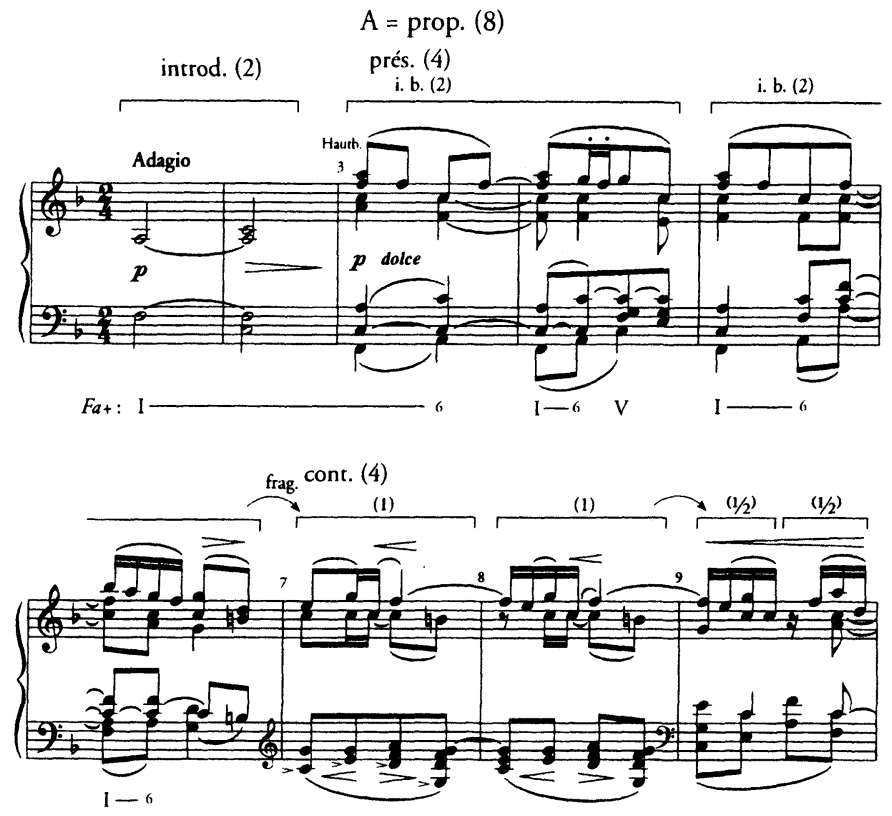

Do+: IV-6 V $\quad$ I-6 ii $^{7} \quad \mathrm{~V}^{7} \quad \mathrm{I}-6 \mathrm{ii}^{7} \quad \mathrm{~V}^{7} \quad \mathrm{I}-6 \quad \mathrm{IV}^{6} \mathbf{3}^{5}$

B (4)

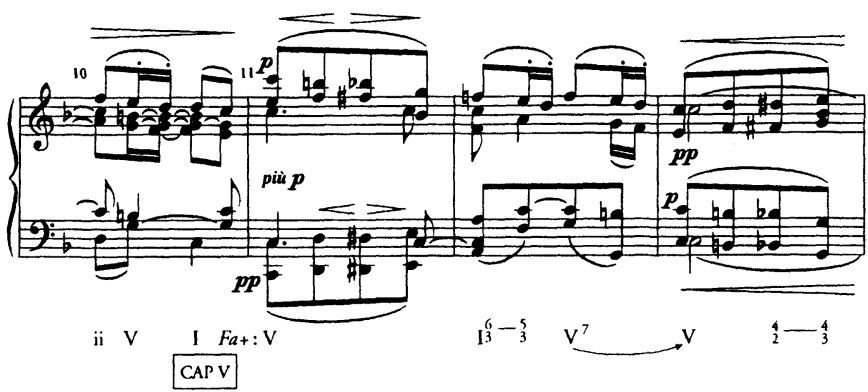

$A^{\prime}(8)$

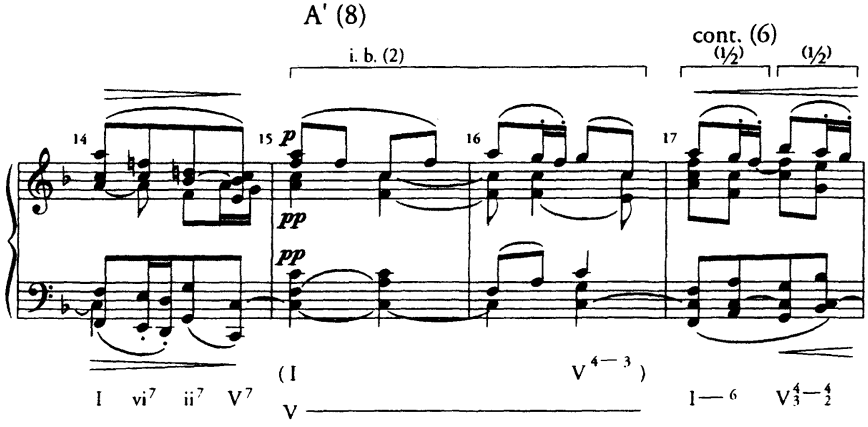

Exemple 2: Brahms, Concerto pour violon en ré majeur, op. $77: 2^{\mathrm{e}}$ mouvement, mes. 1-31 
Exemple 2 - suite

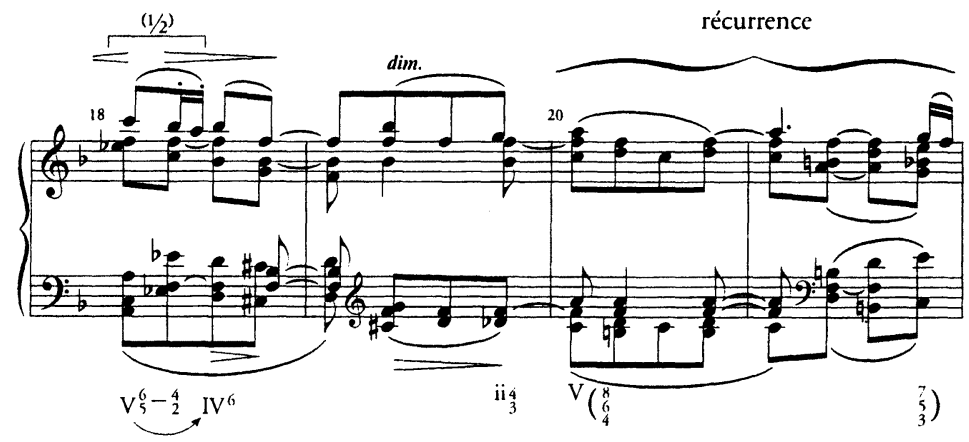

sect. concl. (10) codetta

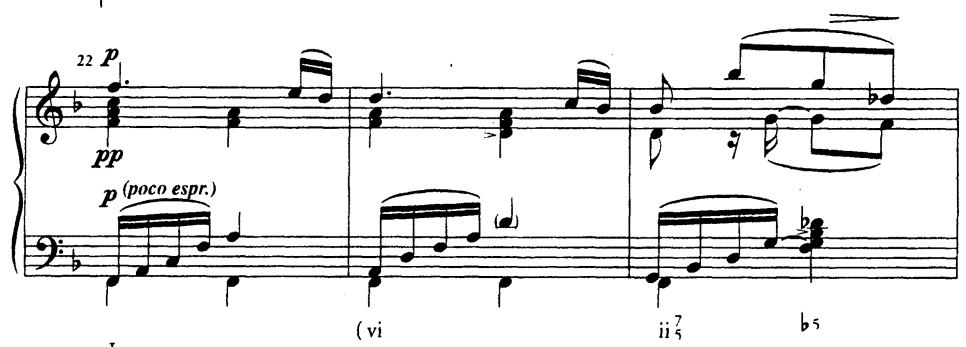
CAPI

cod.

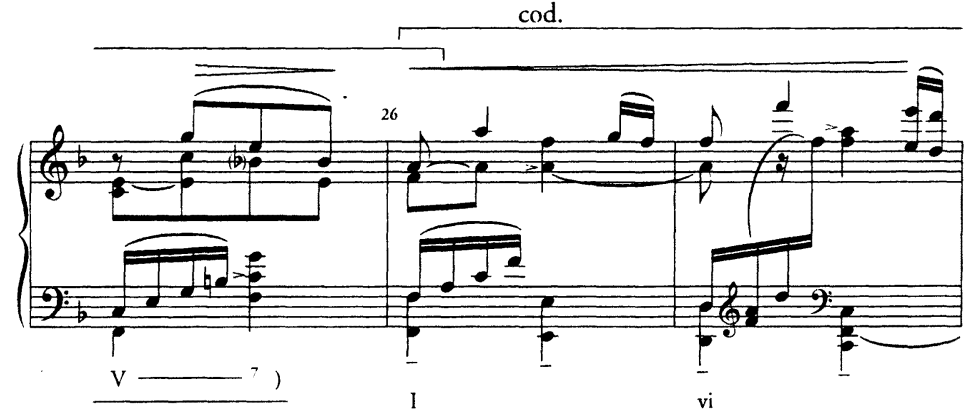

cod. $\Rightarrow$ introd.

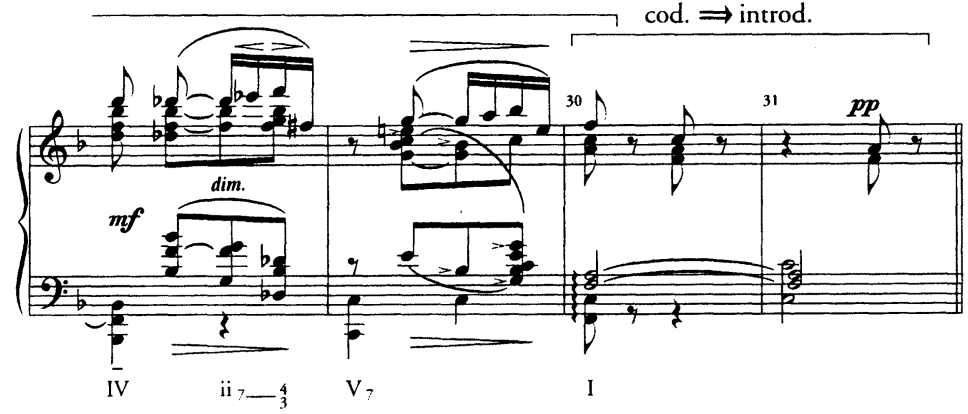


mais en conservant l'idée de base, et en se terminant par une cadence authentique parfaite dans le ton de la tonique.

Dans le thème de Brahms, la section $A$ se termine par une cadence authentique parfaite dans le ton de la dominante, $B$ présente une station sur la dominante, et $\mathrm{A}^{\prime}$ conclut par une cadence authentique parfaite dans le ton de la tonique. L'exposition adopte souvent la forme d'une période, mais celle d'une "proposition ${ }^{9}$, d'une forme « hybride », ou d'un patron non conventionnel sont également possibles. Ici, il s'agit d'une proposition. Bien que cette forme ne constitue pas une structure à retour proprement dite, la fréquence de son imbrication au sein de la petite forme ternaire ou de la période justifie d'en donner une brève description.

Dans sa forme idéale, la proposition est constituée de deux phrases de quatre mesures exprimant trois fonctions formelles différentes, celles de "présentation », " continuation » et de cadence. La fonction de présentation occupe la première phrase et consiste en une idée de base de deux mesures qui fait l'objet d'une répétition. La répétition n'a pas besoin d'être exacte, elle peut aussi s'effectuer en séquence ou selon une réponse tonale (c'est-à-dire une version de l'idée de base sur la dominante, où l'harmonie de tonique est généralement remplacée par celle de dominante et celle de dominante par celle de tonique $)^{10}$. De manière générale, la présentation dans son entier prolonge la tonique. La seconde phrase, appelée continuation pour des raisons de simplification, combine en fait les fonctions de continuation et de cadence. La continuation se manifeste au début de la seconde phrase par un ou plusieurs des traits suivants : la " fragmentation » (qui désigne la diminution de la longueur des groupements de base) ${ }^{11}$, l'augmentation de l'activité rythmique de surface, l'accélération du rythme harmonique et l'usage de séquences harmoniques. Une cadence conclut la phrase.

Dans l'exemple du Concerto de Brahms, la première phrase présente une idée de base de deux mesures (mes. 3-4) répétée avec de légères modifications. Le début de la seconde phrase exprime la fonction de continuation par de la fragmentation (les unités de deux mesures de la présentation sont suivies successivement d'unités d'une mesure aux mes. 7-8, puis d'une demi-mesure à la mes. 9), l'accélération du rythme harmonique (mes. 7 et suivantes), l'augmentation de l'activité rythmique de surface mes. 9 (les doubles croches deviennent plus abondantes), et elle se termine par une cadence authentique parfaite dans le ton de la dominante à la mes. 10 .

\footnotetext{
${ }^{9}$ Les termes Satz en allemand et sentence en anglais sont utilisés respectivement par Ratz, Einführung in die musikalische Formenlehre, et Schönberg, Fundamentals of Musical Composition (Boston : Faber, 1970). Dans la version française de ce dernier ouvrage (Paris : J.-C. Lattès, 1987), Dennis Collins traduit ce terme par «phrase », l'équivalent le plus proche. Ici, Satz est traduit par " proposition ». En grammaire, ce mot désigne une unité syntaxique complète en elle-même (avec un sujet, un verbe et un complément), qui peut être utilisée seule ou en combinaison avec d'autres segments pour former un énoncé plus complexe. L'analogie grammaticale est appropriée, car la proposition musicale forme parfois à elle seule un thème principal, et elle peut être utilisée comme section A d'une petite forme ternaire dans une section thématique.

${ }^{10}$ Voir Caplin, Classical Form, 37-38.

${ }^{11} \mathrm{La}$ «fragmentation » est une traduction de la notion d'Abspaltung chez Ratz, Einführung in die musikalische Formenlehre, ou de fragmentation en anglais chez Caplin, Classical Form.
} 

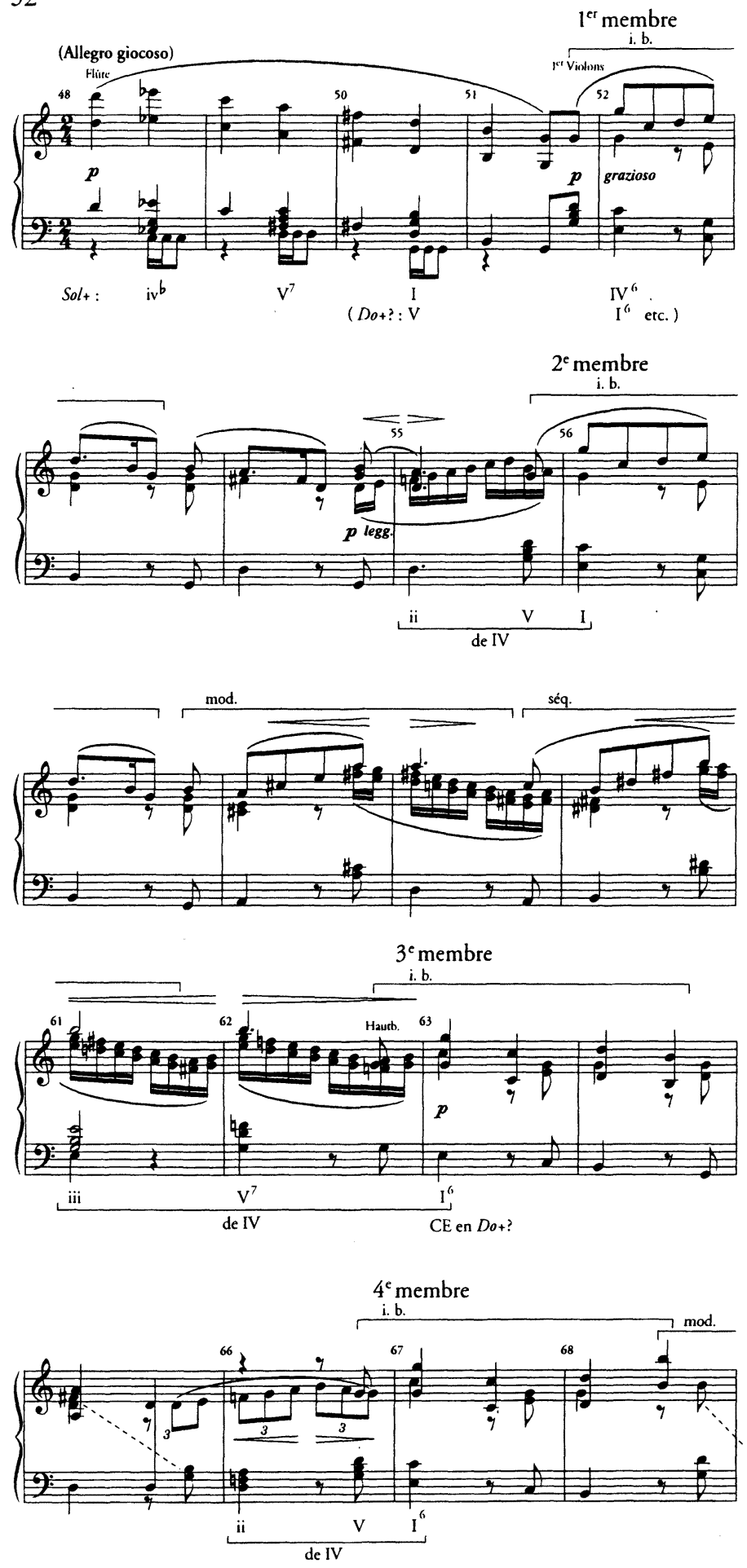

Exemple 3 : Brahms, Symphonie $n^{\circ} 4$ en mi mineur, op. $98: 3^{\mathrm{e}}$ mouvement, mes. 48-90 
Exemple 3 - suite
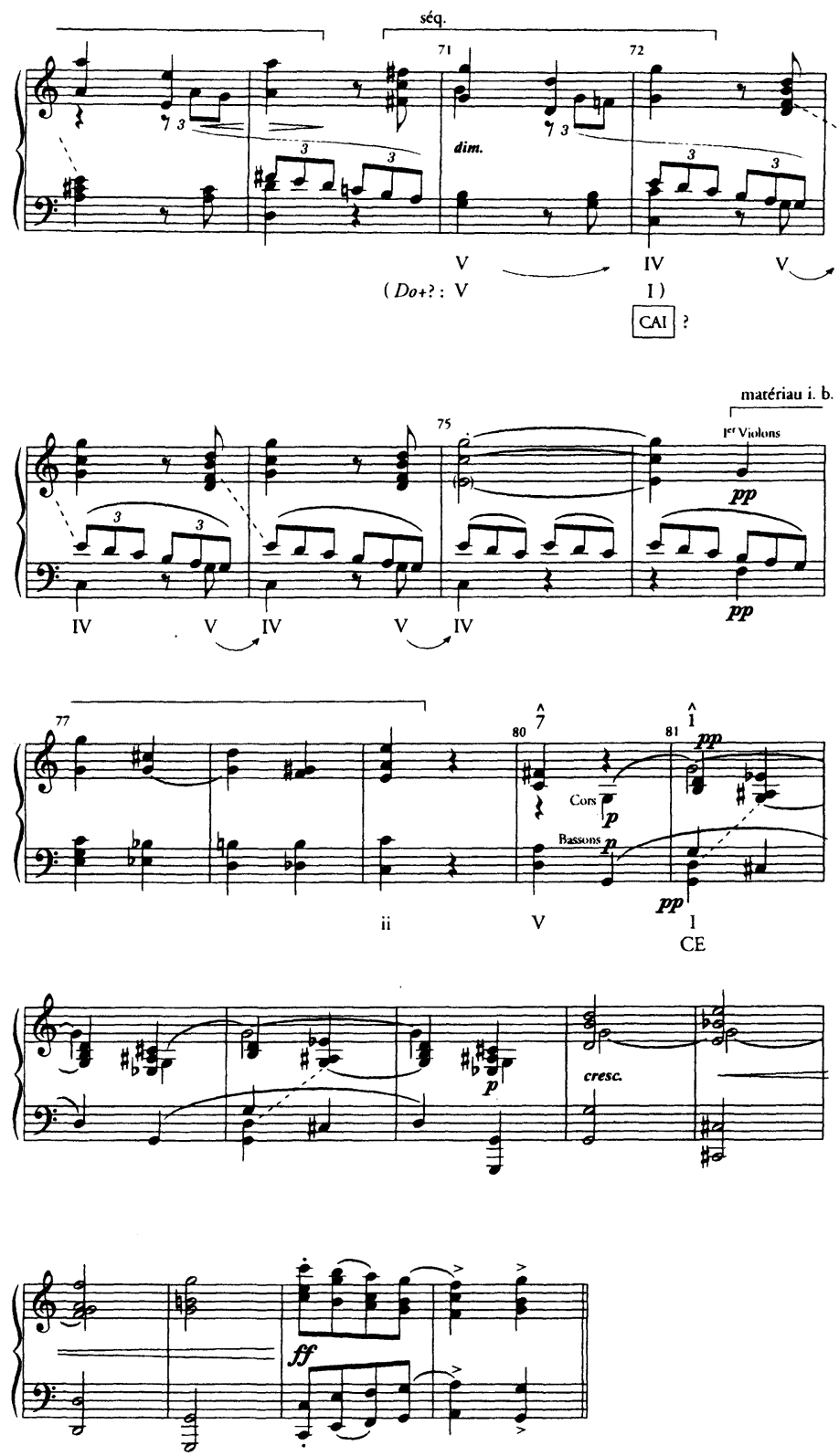
La section $\mathrm{A}^{\prime}$ reprend le matériel de l'exposition avec des modifications considérables. Elle débute par une idée de base unique, suivie d'une continuation allongée. Ces changements n'offrent rien de bien inhabituel pour la forme classique; plusieurs petites formes ternaires de mouvement lent chez Beethoven font d'ailleurs appel au même procédé. Par exemple, le deuxième mouvement de la Sonate pour piano en mi bémol majeur, op. 7, présente aux mes. 1-24 une petite forme ternaire, dont la section $\mathrm{A}^{\prime}$ débute par le retour de l'idée de base aux mes. 15-16 et se poursuit par une continuation. La cadence est suivie d'une « section conclusive » qui ne fait pas partie intégrante de la petite forme ternaire, mais constitue une extension postcadentielle. La section conclusive suit toujours une cadence authentique. Comme c'est le cas ici, elle débute parfois en élision avec la fin de la section précédente et prolonge la tonique par différents moyens, comme l'utilisation d'une pédale de tonique (mes. 22-26), de formules cadentielles (sur la pédale de tonique aux mes. 22-26, puis sans pédale mes. 2630 ), et d'accords de tonique répétés (mes. 30-31).

Si les deux thèmes de Brahms illustrant la période et la petite forme ternaire se conforment étroitement au modèle classique, la théorie de Caplin s'avère impuissante à rendre compte de la variété d'organisations des structures à retour de Brahms. Le thème subordonné du troisième mouvement de la Symphonie $\mathrm{n}^{\circ} 4$ en mi mineur, op. 98 (mes. 52-81, exemple 3 ) offre une illustration de structure fondamentalement irréductible aux types formels classiques. Les multiples reprises du matériau de l'idée de base, exprimant toujours la même fonction de début, de commencement, permettent de ranger cette vaste section de 30 mesures dans la catégorie des structures à retour, mais le patron formel et l'organisation structurelle du thème se distinguent radicalement des principes classiques.

La disposition des retours dans cet extrait produit une segmentation claire. L'idée de base par laquelle débute le thème (identifiée par un crochet aux mes. 52-53) fait l'objet de trois retours, une fois par reprise littérale aux mes. 56-57, et les deux autres fois de manière variée, aux mes. 63-64 et 67-68. L'utilisation du matériau de l'idée de base au moment de la cadence finale (mes. 77-78) ne constitue pas un véritable retour, car le matériau n'exprime pas la même fonction de début, mais joue un rôle conclusif. La reprise de la cellule initiale définit donc une structure en quatre membres inégaux de 4,7,4 et 15 mesures aux mes. $52-55,56-62,63-66$ et $67-81$ (la figure 1 propose une représentation schématique de la forme).

Un trait fondamental du thème - son ambiguïté tonale - est intimement lié à l'organisation cadentielle. La section est présentée en sol majeur, dans le ton de la dominante, comme il est d'usage pour le thème subordonné d'une forme sonate de tonalité majeure, mais le contraste entre ton principal et subordonné est ici affaibli par l'accent exceptionnel mis sur do majeur (la sousdominante du ton subordonné, soit le ton principal du mouvement).

L'ambiguité entre les deux tons, perceptible dès le début du thème, est renforcée par l'organisation des fonctions conclusives : on ne relève aucun geste cadentiel en sol avant celui qui marque la fin du thème, la cadence évitée (CE) de la mes. 81. Ce geste cadentiel est inabouti à cause de l'absence de résolution de la sensible de la mélodie (degré 7), présentée dans la partie des premiers violons (mes. 80). La 
partie de cor amène bien au début de la mesure suivante le degré attendu (1), et qui plus est, dans le registre approprié, mais cet instrument double en fait le début de la ligne mélodique d'une nouvelle phrase exposée aux bassons.

\begin{tabular}{|c|c|c|c|}
\hline & $\begin{array}{l}\text { partie (11) } \\
\text { s. 52-62 }\end{array}$ & & $\begin{array}{l}\text { e (19) } \\
3-81\end{array}$ \\
\hline $1^{\mathrm{er}}$ membre (4) & $2^{e}$ membre $(7)$ & $3^{\mathrm{e}}$ membre (4) & $4^{e}$ membre $(15)$ \\
\hline
\end{tabular}

Cadence :

$$
\begin{gathered}
\mathrm{CE} \\
\text { en } d o+?
\end{gathered}
$$

63

Mesure :

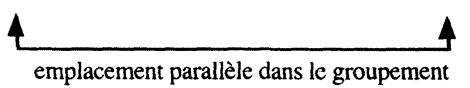

$\begin{array}{cc}\text { CIA } & \text { CE } \\ \text { en } d o+? & \text { en sol+ }\end{array}$

$72 \quad 81$

Figure 1 : Brahms, Symphonie $n^{\circ} 4$ en mi mineur, op. 98 : $3^{\mathrm{e}}$ mouvement, mes. 52-81

On peut donc observer plusieurs entorses aux principes classiques. D'abord, la combinaison particulière des cadences et des retours produit un patron non conventionnel, inconnu des classiques. De plus, les classiques font toujours suivre le retour de l'idée de base au début du conséquent ou de la section $\mathrm{A}^{\prime}$ d'une cadence authentique. Le thème de Brahms ne produit de geste cadentiel clair qu'à la suite du dernier retour, et encore s'agit-il d'une cadence évitée et non d'une cadence authentique (mes. 81); l'absence de cadence suivant le retour des deux membres intérieurs marque donc un écart supplémentaire par rapport à la pratique classique.

Les irrégularités ne se limitent pas à la combinaison des cadences et des retours, mais affectent la manipulation même de ces éléments de base. L'articulation de la fin d'un thème subordonné par une cadence évitée contredit les principes classiques. Ceux-ci prescrivent l'utilisation de la cadence évitée pour retarder l'arrivée de la cadence, et requièrent l'usage d'extensions jusqu'à ce qu'un geste cadentiel abouti se produise ${ }^{12}$. Le thème de Brahms exploite aussi des effets cadentiels inconnus de la catégorisation cadentielle classique, comme l'esquisse d'un geste cadentiel dans une tonalité ambiguë à la mes. 63 . Finalement, les variations de tension du thème acquièrent une importance inusitée dans la définition de l'effet cadentiel. Chez les classiques, les fluctuations d'intensité survenant au moment des cadences se limitent à confirmer la fonction conclusive. Chez Brahms et les romantiques, elles jouent un rôle structurel plus important et peuvent influencer la perception de l'effet cadentiel en clarifiant un geste ambigu ou, au contraire, en créant des fausses attentes.

12 Voir William Caplin, « Structural Expansion in Beethoven's Symphonic Forms », dans Beethoven's Compositional Process, édité par William Kinderman (Lincoln :University of Nebraska Press, 1991), 32; et Janet Schmalfeldt, "Cadential Processes : The Evaded Cadence and the "One More Time" Technique », Journal of Musicological Research 12 (1992) : 1-2. 
La redéfinition de l'organisation des cadences et des retours dans certains thèmes à retour de Brahms, comme dans celui de l'exemple 3 , prouve la nécessité d'intégrer ces paramètres dans l'élaboration d'un modèle formel alternatif. En guise de préliminaire à une telle entreprise, voici maintenant une étude de l'utilisation du retour et de l'organisation cadentielle.

\section{LE TRATTEMENT DU RETOUR CHEZ BRAHMS ET LES CLASSIQUES}

Trois catégories de retour thématique sont utilisées dans les œuvres des classiques et de Brahms : les retours « exacts », « séquentiels ", et "par réponse tonale $»^{13}$. La petite forme ternaire emploie exclusivement le retour exact, mais les trois types sont admissibles dans la période et les structures dérivées. Dans le retour exact, qui est illustré par l'ensemble des exemples cités jusqu'à maintenant, l'idée de base initiale est généralement reprise sur les mêmes hauteurs, bien qu'il ne soit pas nécessaire qu'elle réapparaisse de façon identique. Par exemple, elle peut être transposée dans un registre différent. Ainsi, le thème initial du mouvement lent de la Symphonie $\mathrm{n}^{\circ} 1$ en do mineur de Brahms, op. 68 , présente d'abord l'idée de base à la mélodie aux mes. 1-2, et la transfère à la basse lors du retour aux mes. 22-23 (la section médiane du même mouvement reprend le même procédé aux mes. 39-40 et 47-48). Dans d'autres cas, le matériau de l'idée de base est ornementé au moment du retour, comme dans la variation par hétérophonie de l'idée de base du thème initial du troisième mouvement du Quatuor à cordes en si bémol majeur, op. 67 (comparer les mes. 13-14 à l'alto et le premier violon avec l'alto aux mes. 1-2), mais le support harmonique reste identique.

Le retour séquentiel désigne un retour sur tout autre degré que I ou V, et se produit généralement sur ii chez les classiques. Ce procédé est illustré dans le thème principal de la Sonate pour piano en ré majeur, K.576, de Mozart. Le thème est constitué d'une période de huit mesures. L'idée de base de l'antécédent, qui est entièrement fondée sur une harmonie de tonique aux mes. 1-2, est reprise sur une harmonie de sus-tonique au début du conséquent aux mes. 5-6 $6^{14}$.

L'utilisation des retours séquentiels chez les romantiques ne se limite pas aux reprises de l'idée de base sur ii, comme c'est généralement le cas chez les classiques, et l'élargissement de l'éventail des transpositions témoigne d'une expansion de l'espace tonal semblable à celle qui a été observée dans l'organisation cadentielle. Cette prospection débute déjà avec Beethoven, qui utilise par exemple un retour sur bII dans le thème principal de la Sonate pour piano en fa mineur, op. 57 ( «Appassionata »). On trouve également des exemples de retours séquentiels dans la musique romantique sur les degrés suivants (cette liste ne prétend pas à l'exhaustivité) :

${ }^{13}$ Caplin, Classical Form, 53.

${ }^{14}$ On trouvera un exemple de retour séquentiel emprunté au thème principal du Trio avec piano $\mathrm{n}^{\circ} 1$ en si bémol majeur, D. 898, de Schubert, dans Leonard B. Meyer, Style and Music : Theory, History, and Ideology (Philadelphie : University of Pennsylvania Press, 1989), 230-31. L'auteur suggère que ce procédé caractéristique des romantiques est inconnu des classiques. L'extrait de la Sonate pour piano en ré majeur, K. 576, de Mozart apporte la preuve du contraire. 
1. bIII, dans le thème initial du second mouvement de la Sonate pour piano $\mathrm{n}^{\circ} 3$ en fa mineur, op. 5, de Brahms.

2. IV, dans les thèmes principaux de la Symphonie $\mathrm{n}^{\circ} 1$ en si bémol majeur, op. 38, de Schumann et du Concerto pour violoncelle en si mineur, op. 104, de Dvorák et, chez Brahms, dans le refrain du finale du Concerto pour piano $\mathrm{n}^{\circ} 1$ en ré mineur, op. 15 , et le thème initial du mouvement lent de la Sonate pour violoncelle $\mathrm{n}^{\mathrm{0}} 2$ en fa majeur, op. $99^{15}$.

3. vi, dans le thème initial du second mouvement du Quatuor avec piano $\mathbf{n}^{\circ}$ 2 en la majeur, op. 26, de Brahms.

4. bVII ou bvii, respectivement dans le refrain du finale du Concerto pour deux pianos $\mathrm{n}^{\circ} 2$ en la bémol majeur de Mendelssohn, et dans le thème principal du Quatuor avec piano $\mathrm{n}^{\circ} 3$ en do mineur, op. 60, de Brahms ${ }^{16}$.

Certains compositeurs romantiques comme Chopin affichent une véritable prédilection pour les retours séquentiels, peut-être pour l'effet dramatique de tonicisation des régions mineures qu'ils produisent souvent. Chez Brahms, cependant, ce type de retour n'est pas aussi fréquent que le retour exact. Un exemple de retour séquentiel sur ii très similaire à celui de Mozart se produit dans le thème initial du Trio avec piano $\mathrm{n}^{\circ} 1$, op. 8, à la mes. 25 (exemple 4). Le thème est construit selon une petite forme ternaire dont les sections $B$ et $A^{\prime}$ font l'objet d'une répétition variée, ce qui produit un patron $A B A^{\prime} B^{\prime} A^{\prime \prime}$, et le thème se termine par un geste cadentiel inhabituel (mes. 44). La forte redondance du matériau thématique dans cette forme est atténuée par un procédé typiquement haydnesque consistant à utiliser le retour séquentiel pour varier la présentation de l'idée de base ${ }^{17}$. La section $\mathrm{A}$ est construite sur une période

${ }^{15}$ Cité par Margaret Notley, « Brahms's Cello Sonata in F Major and Its Genesis : A Study in Half-Step Relations ", dans Brahms Studies, Volume 1, édité par David Brodbeck (Lincoln : University of Nebraska Press, 1994), 139-60. Dans certains cas, le retour sur IV pourrait s'interpréter comme un retour par réponse tonale. Dans une présentation, l'idée de base fondée sur le mouvement de $\mathrm{I}-\mathrm{V}$, reprise avec des variantes de manière à produire la progression IV-I, constitue une forme de répétition par réponse tonale. Par analogie, le retour sur IV est considéré comme un retour par réponse tonale dans les cas où le conséquent produit un mouvement de IV-I contrebalançant le mouvement de I-V de l'antécédent. C'est ce qui se produit dans le thème initial du Faschingsschwank aus Wien, op. 26, de Schumann, ou chez Beethoven dans les thèmes initiaux du finale de la Sonate pour violon en la majeur, op. $12, \mathrm{n}^{\circ} 2$, et du troisième mouvement de la Sonate pour piano en mi majeur, op. 109. Mais, dans les exemples cités plus haut, aucun des retours sur IV n'est suivi d'un mouvement vers la tonique, et il est donc préférable de parler de retour séquentiel.

${ }^{16}$ Arno Mitschka relève également un retour surbVII dans le thème principal de la Sonate pour piano $\mathrm{n}^{\circ} 1$ en do majeur de Brahms, op. 1, mais sa comparaison avec la répétition séquentielle de l'idée de base sur bVII dans le thème principal de la Sonate pour piano en do majeur, op. 53 ( "Waldstein »), de Beethoven suggère qu'il ne fait pas vraiment la distinction entre les procédés de répétition et de retour. Voir «Der Sonatensatz in den Werken von Johannes Brahms » (thèse de doctorat, Johannes-Gutenberg-Universität, Mainz, 1961).

${ }^{17}$ Ceci est illustré par exemple chez Haydn dans le refrain du finale de la Symphonie n ${ }^{\circ} 96$ ( « Le miracle »). 
Exemple 4 : Brahms, Trio n 1 en si majeur, op. 8 (rév. 1889), mes. 1-44
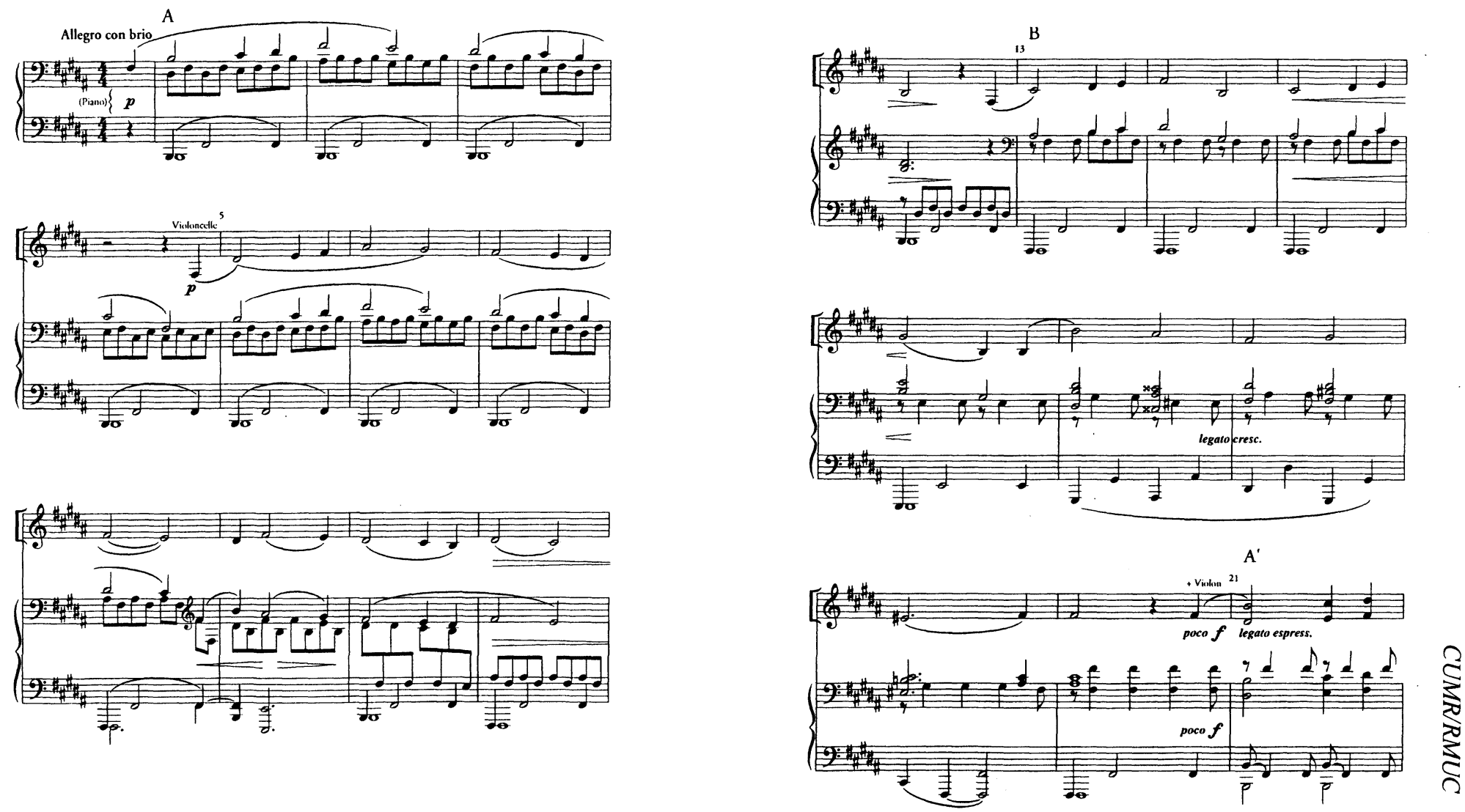

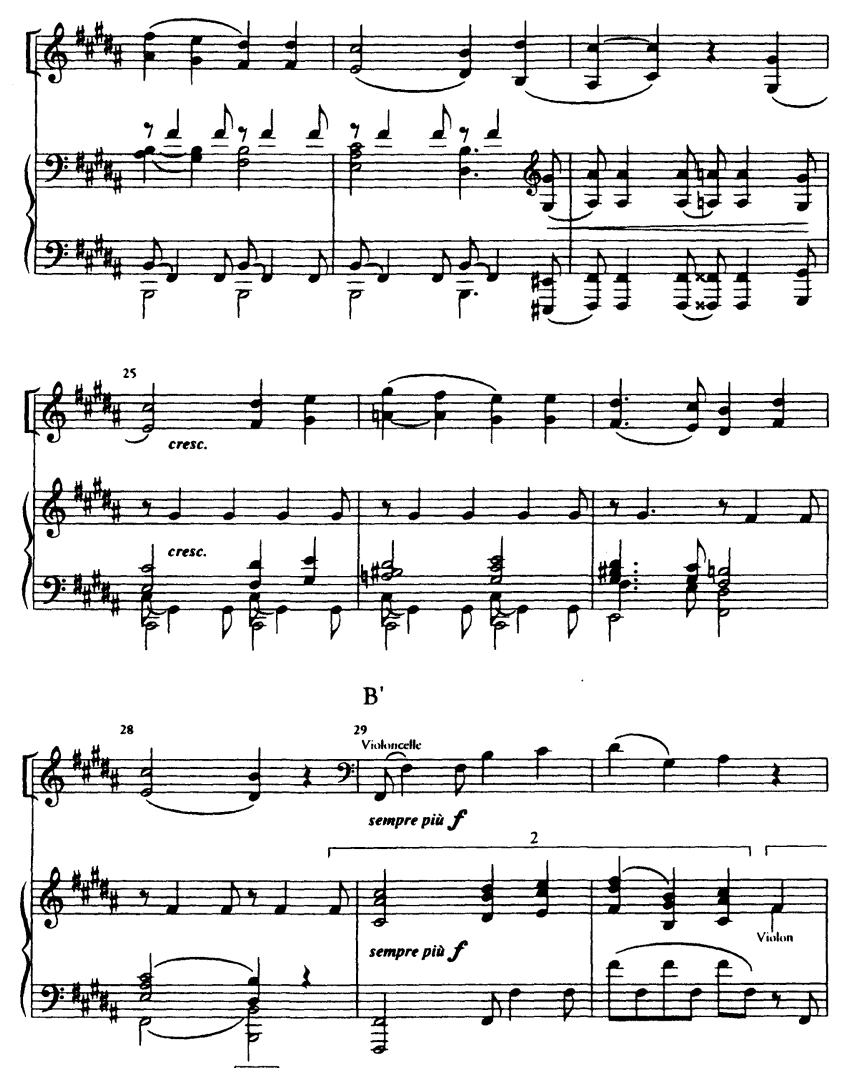

CAP

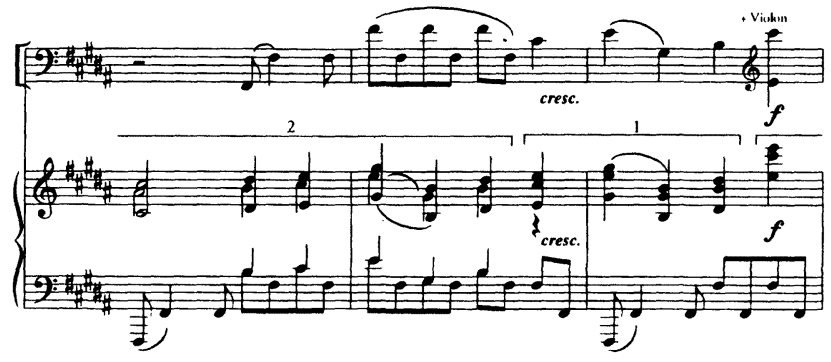

$\frac{N}{N}$

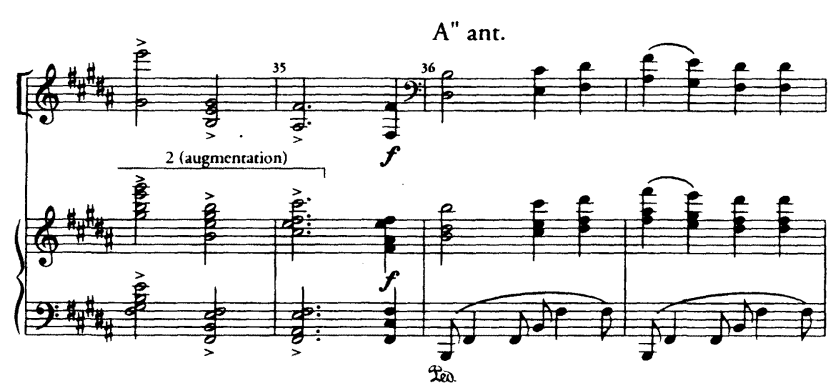


Exemple 4 - suite
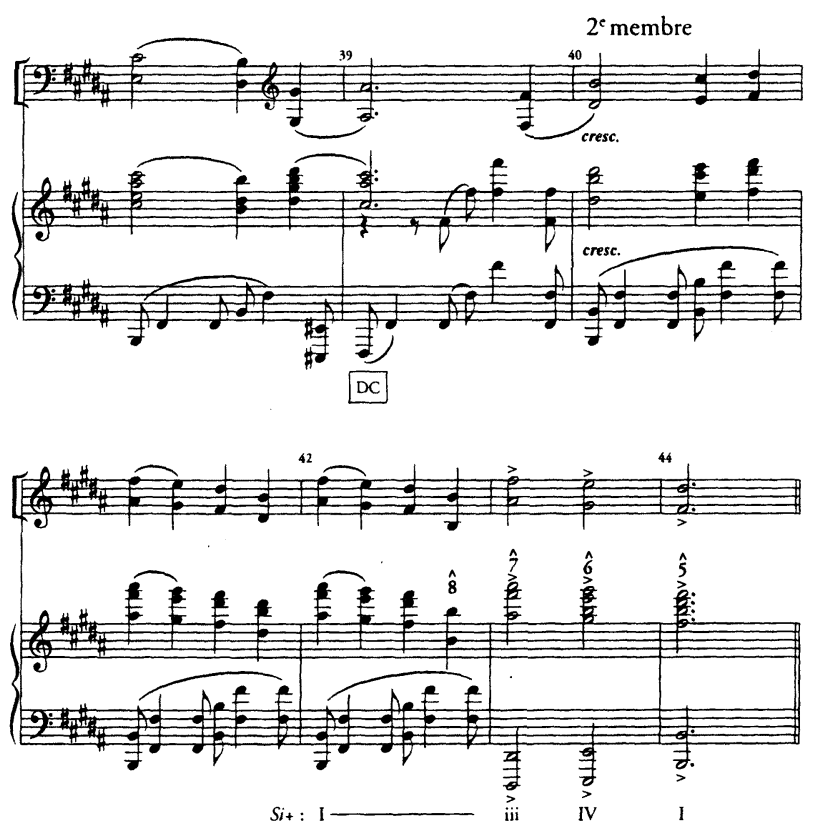

offrant un retour exact de l'idée de base à la mes. 5; l'harmonie reste identique, mais la mélodie est variée par contrepoint renversable, la série de sixtes parallèles étant renversée en tierces. L'idée de base de l'antécédent de A' (mes. 21-22) fait l'objet d'un retour séquentiel sur ii au début du conséquent (mes. 25-26).

Dans le retour par réponse tonale, le conséquent débute par une version de l'idée de base initiale sur la dominante, de manière à ce que le mouvement harmonique général de l'antécédent I-V soit contrebalancé par le mouvement inverse dans le conséquent, $\mathrm{V}-\mathrm{I}$. Ce procédé est illustré dans la période qui ouvre le thème principal du premier mouvement de la Symphonie $n^{\circ} 96 \mathrm{de}$ Haydn («Le miracle »). L'antécédent commence sur la tonique à la mes. 18 et se termine par une demi-cadence à la mes. 21. Le conséquent reprend l'idée de base dans une version sur la dominante aux mes. 22-23 et conclut sur la tonique par une cadence authentique parfaite à la mes. 25 .

Le retour par réponse tonale, relativement rare chez Brahms, est illustré dans le Quatuor avec piano $\mathrm{n}^{\circ} 1$ en sol mineur, op. 25. Le thème principal adopte un patron de petite forme ternaire, et une période s'imbrique dans la section $\mathrm{A}$ aux mes. 1-10 (exemple 5). L'antécédent débute sur la tonique et se termine par une cadence sur la dominante à la mes. 4 . Le conséquent reprend aux mes. 5-6 les deux premières mesures de l'antécédent, transposées sur la dominante mineure, et se termine par une cadence authentique parfaite dans le ton principal. Les deux membres de la période produisent donc les deux mouvements harmoniques successifs $\mathrm{i}-\mathrm{V}, \mathrm{v}-\mathrm{i}$. 

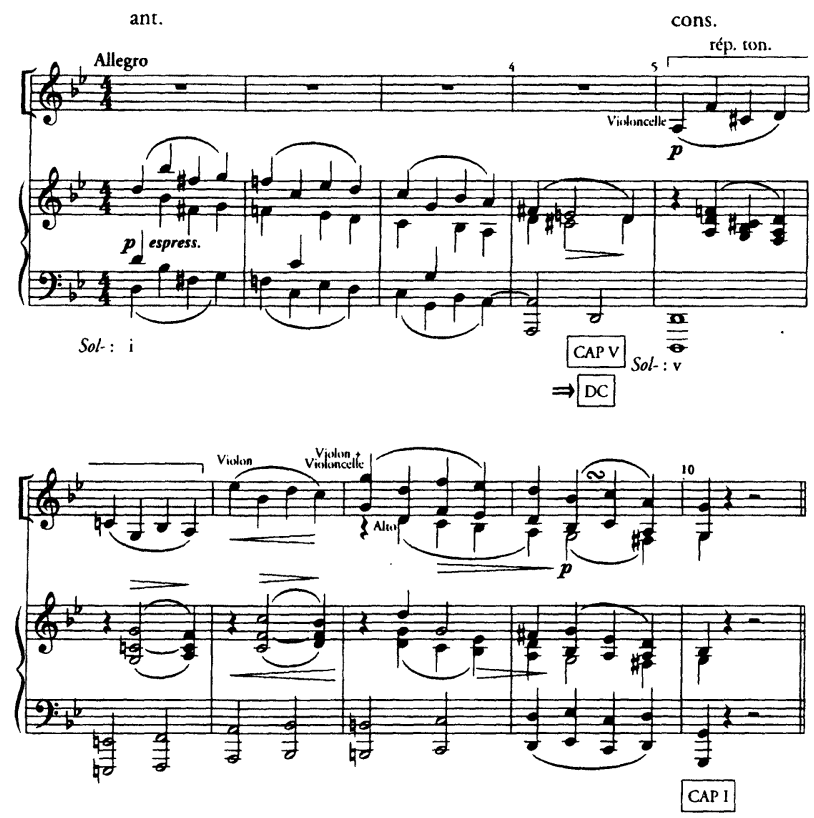

Exemple 5 : Brahms, Quatuor avec piano $\mathrm{n}^{\circ} 1$ en sol mineur, op. $25: 1^{\mathrm{er}}$ mouvement, mes. 1-10

L'utilisation de la réponse tonale chez Brahms présente une différence fondamentale par rapport à celle des classiques. Dans le thème de Haydn cité plus haut, le mouvement d'éloignement de la tonique vers la dominante dans un premier membre de quatre mesures est compensé par le mouvement inverse de la dominante vers la tonique dans les quatre mesures suivantes. Ainsi, la symétrie harmonique produite par le retour par réponse tonale (I-V par opposition à V-I) est renforcée par la symétrie de groupement des deux groupes de quatre mesures. L'exemple de Brahms brise à la fois la symétrie harmonique et celle du groupement. L'utilisation de l'accord de dominante mineure à la place d'un accord majeur au moment du retour détruit la symétrie harmonique. Cette substitution a pour effet de poursuivre le mouvement d'éloignement de la tonique amorcé dans l'antécédent, et donc de retarder le moment de résolution. C'est comme si l'accord de $\mathrm{v}$, qui témoigne d'un éloignement harmonique accru par rapport à la tonique en comparaison avec l'accord de $\mathrm{V}$ (majeur), nécessitait un allongement du second membre (qui fait six mesures, soit deux mesures de plus que l'antécédent) pour permettre d'effectuer le mouvement de retour vers la tonique ${ }^{18}$.

${ }^{18}$ Des exemples de retour sur un accord de $\mathbf{v}$ mineur se produisent également dans la littérature classique, comme aux mes. 9-10 du premier mouvement de la Sonate pour piano en fa mineur, op. $2, \mathrm{n}^{\circ} 1$, de Beethoven. Malgré les apparences, il ne s'agit pas d'un véritable retour par réponse 
Le rejet de la double symétrie classique chez Brahms ne se limite pas au retour sur la dominante mineure, mais s'observe également dans le retour par réponse tonale sur une dominante majeure. C'est le cas du thème initial du trio de l'«Intermezzo » du Quatuor en sol mineur, op.25 (mes. 117-31), une structure à retour composée de trois membres. Après le mouvement I-V de l'antécédent (mes. 117-22), le mouvement traditionnel de V-I est distribué sur les deux derniers membres : V-bVI et bVI (en passant par V)-I (successivement aux mes. 123-27 et 128-31).

Le rejet de la double symétrie dans les retours par réponse tonale peut s'expliquer de la manière suivante. Les repères fournis à l'auditeur par les conventions assurent une certaine prévisibilité structurelle. Par exemple,l'utilisation d'une demi-cadence à la fin d'un antécédent suggère déjà la possibilité d'un conséquent. Le degré de prévisibilité augmente considérablement dans le répertoire classique lorsque le conséquent débute par un retour par réponse tonale. L'auditeur est presque certain de pouvoir anticiper une cadence sur la tonique terminant le second membre dans un emplacement parallèle avec la cadence du premier membre. Le rejet de la double symétrie classique dans l'utilisation du retour par réponse tonale chez Brahms peut donc être interprété comme une façon d'éviter une trop grande prévisibilité.

Les implications cadentielles particulières du retour dans les thèmes de Brahms (et ceux des romantiques en général) permettent d'expliquer l'émergence de nouveaux patrons formels. La reprise de l'idée de base dans le conséquent ou la section $\mathrm{A}^{\prime}$ chez les classiques est généralement suivie d'une cadence authentique. On compte bien quelques exceptions, comme la période renversée, dont le premier membre se termine par une cadence authentique parfaite et le retour est suivi d'une demi-cadence, mais elles sont plutôt rares. L'imbrication d'une période au sein d'une section $A^{\prime}$ représente un cas particulier : le retour qui signale le début de la section $A^{\prime}$ est séparé de la cadence authentique parfaite par une demi-cadence et un autre retour de l'idée de base marquant respectivement la fin de l'antécédent et le début du conséquent. Mais, dans ce cas-ci, le retour de l'idée de base au début du conséquent se situe à un niveau structurel inférieur à celui de la section $\mathrm{A}^{\prime}$, et la cadence authentique parfaite finale constitue le but, le point d'aboutissement des deux.retours. Contrairement aux classiques, la reprise de l'idée de base dans le conséquent ou la section $\mathrm{A}^{\prime}$ n'est pas toujours suivie d'une cadence authentique chez Brahms et les romantiques. La tendance à retarder l'arrivée de la cadence finale les conduit parfois à intercaler une ou plusieurs faibles cadences intermédiaires (généralement des demi-cadences) entre le retour et la cadence authentique, ou même à éliminer complètement la cadence

tonale. Le thème principal présente une structure de proposition et ressemble à un antécédent parce qu'il se termine par une demi-cadence et que l'idée de base est reprise tout de suite après. Mais le segment qui débute par le retour constitue la transition, et il produit la modulation au tonsubordonné. L'utilisation de l'accord $\mathrm{v}$ au début de la transition est parfaitement adapté à cette fonction formelle, car il contribue à la déstabilisation de la tonique et l'effet de renforcement du mouvement d'éloignement de la tonique donne une impulsion à la modulation au ton subordonné. 
Double hybride (28)

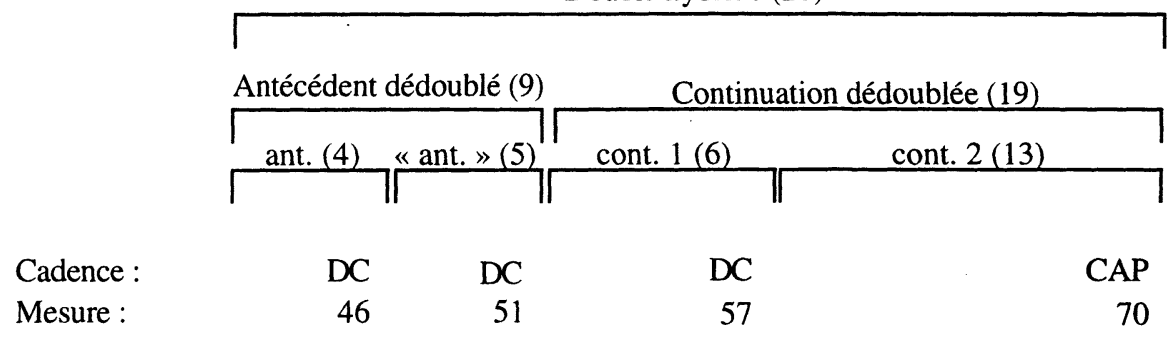

Figure 2 : Brahms, Symphonie $\mathrm{n}^{\circ} 1$ en do mineur, op. $68: 1^{\text {er }}$ mouvement, mes. 43-70

authentique $^{19}$. Dans le thème principal de la Symphonie $\mathrm{n}^{\circ} 1$ en do mineur, op. 68 (analysé sous une forme schématique à la figure 2), le retour de l'idée de base (mes. 47-48) suit une demi-cadence (mes. 46), et est séparé de la cadence authentique finale (mes. 70) par deux demi-cadences (mes. 51 et 57). Les cadences définissent une structure en quatre membres, aux mes. 43-46,47-51,5257 et $58-70$.

Le premier membre présente une structure d'antécédent typique de quatre mesures, composé d'une idée de base et d'une idée contrastante de deux mesures chacune, et se terminant par une demi-cadence à la mes. 46 . Le second membre qui présente une expansion par rapport au premier débute par un retour par réponse tonale de l'idée de base (sur v), et se termine par une autre demicadence à la mes. 51, plus appuyée que la première. La fonction formelle de ce segment est très particulière : il ne s'agit pas d'un conséquent, car il se termine par la même cadence que le premier membre. Mais ce n'est pas une répétition de l'antécédent non plus, car le membre est différent, plus long, et le sens de véritable retour est renforcé par la transposition de l'idée de base. Il s'agit plutôt d'un « second antécédent ». Les deux membres suivants expriment la fonction de continuation par la fragmentation (qui produit des segments d'une mesure) et l'instabilité harmonique. Le matériau qui ouvre la première continuation (mes. 52-55) fait l'objet d'une reprise séquentielle au début de la seconde (mes. 58-61). L'organisation formelle des membres suggère une structure bipartite regroupant ensemble les deux antécédents d'une part et les deux parties de continuation d'autre part. La combinaison des fonctions d'antécédent et de continuation évoque un type d'hybride, mais dont chaque partie aurait été dédoublée.

Le thème joue sur l'attente d'une cadence parfaite, dont l'arrivée est différée le plus longtemps possible. D'après les conventions classiques, l'utilisation d'un retour après un antécédent rend très probable l'arrivée d'une cadence authentique. Les attentes par rapport à l'arrivée d'une cadence authentique parfaite, déçues par l'utilisation d'une demi-cadence à la fin du double

${ }^{19} C^{\prime}$ est le cas du thème de la Symphonie no 4 en mi mineur, op. 98, cité à l'exemple 3. 
antécédent, renforcées par la récurrence séquentielle de matériau dans la continuation, sont finalement satisfaites à la fin du thème. Le processus d'expansion de la longueur des segments (passant successivement de 4 à 5 , à 6 , puis à 13 mesures) renforce l'impression que l'arrivée de la cadence est retardée. L'effet d'expansion est d'autant plus frappant que chaque segment est dédoublé; le premier terme du couple sert donc d'étalon pour mesurer l'expansion du second terme.

D'autres structures à retour prennent des libertés similaires avec l'organisation cadentielle et produisent toute une variété de patrons formels. Par exemple, le trio du troisième mouvement de la Sonate pour clarinette en fa mineur, op. 120, $\mathrm{n}^{\circ} 1$, de Brahms présente une forme de période de 16 mesures différente de tous les modèles classiques (figure 3). L'idée de base (mes. 47-48) fait l'objet de trois retours aux mes. 51-52, 55-56 et 59-60. La cadence authentique qui, d'après les conventions classiques, devrait arriver après le premier retour est retardée jusqu'à la mes. 62. Entre le premier retour et la cadence finale s'intercalent une demi-cadence et deux autres retours (le premier est dépourvu d'articulation, et le second est finalement suivi de la cadence). Les idées de base composées («i.b.c.») renvoient à des phrases de quatre mesures qui combinent une idée de base et une idée contrastante sans produire de cadence.

\begin{tabular}{|c|c|c|c|}
\hline \multicolumn{2}{|c|}{ Ant. (8) } & \multicolumn{2}{|c|}{ Cons. (8) } \\
\hline i.b.c. (4) & «ant. » (4) & i.b.c. (4) & cons. (4) \\
\hline i.b. (2) + i.c. (2) & $\|$ i.b. (2) + i.c. (2) $\|$ & i.b. $(2)+$ i.c. (2) & i.b. (2) + i.c. (2) \\
\hline & DC & & CAP \\
\hline 47 & 54 & 55 & 59 \\
\hline
\end{tabular}

Cadence :

Mesure :

47

Figure 3: Brahms, Sonate pour clarinette en fa mineur, op. 120, $\mathrm{n}^{\circ} 1: 3^{\mathrm{e}}$ mouvement (trio), mes. 47-60

La tendance à éviter les cadences fortes est tellement marquée dans certaines structures périodiques que le retour suivant l'antécédent n'est jamais suivi par une cadence authentique. C'est le cas du thème principal du Quintette avec piano en fa mineur, op. 34, de Brahms. Le thème, dépourvu de cadence authentique, est composé de deux grandes sections (mes. 1-11 et 12-22) qui débutent toutes les deux par la même idée de base. Chaque partie semble construite sur une structure hybride, composée d'un antécédent et d'une continuation. L'absence de toute cadence authentique dans ce thème à retour produit à nouveau une forme particulière . La première partie semble devoir jouer le rôle d'antécédent à grande échelle en vertu de sa terminaison sur une demi-cadence précédant le retour de l'idée de base au début du membre suivant, mais l'analyse de la fonction de la seconde partie pose des problèmes similaires à ceux du thème principal de la première symphonie. Malgré le retour de l'idée 
de base, il ne s'agit pas d'un conséquent, car le membre se termine par la même articulation sur $\mathrm{V}$ que la première partie; mais cela ne correspond pas plus à une simple répétition de l'antécédent. Il s'agit donc d'un second antécédent. L'organisation formelle du thème principal du Quintette ne constitue pas un cas isolé. Une structure très similaire se produit dans le thème principal du Quatuor avec piano en do mineur, op. 60. Ces deux thèmes, caractérisés par un souffle dramatique aux accents quasi symphoniques, présentent une structure d'antécédent dédoublé qui évite complètement la cadence parfaite ${ }^{20}$.

Comme on a pu le constater, l'utilisation du retour chez Brahms se situe dans la continuité des classiques. Il utilise les mêmes trois catégories de retour que ses précédesseurs (retour exact, séquentiel et par réponse tonale), mais de manière différente. L'exploration de nouvelles transpositions dans le retour séquentiel témoigne d'une expansion des relations tonales typiquement romantique, et on peut invoquer la tendance à éviter un caractère trop prévisible pour justifier le rejet de la double symétrie classique dans le retour par réponse tonale (principe qui coordonne la symétrie de groupement des deux membres avec la symétrie du mouvement harmonique, I-V et V-I). Finalement, un rapport nouveau aux implications cadentielles du retour permet l'émergence de nouveaux patrons formels chez Brahms. Celui-ci abadonne l'obligation générale chez les classiques de faire suivre le retour par une cadence authentique. La section suivante va montrer comment l'emploi de la cadence témoigne également d'un élargissement de la pratique classique.

\section{L'ORGANISATION CADENTIELLE CHEZ BRAHMS ET LES CLASSIQUES}

Le discours analytique dominant traite généralement la tonalité comme un paramètre défini en termes strictement harmoniques, sans égard pour d'autres dimensions comme le rythme ou le groupement (comme le laisse facilement croire l'étude d'un graphique schenkérien par exemple, bien que le processus analytique soit plus complexe). Notre approche tente de fournir une vision plus juste en accordant une importance déterminante au paramètre formel, et plus particulièrement à l'organisation cadentielle, dans l'articulation de la tonalité (ce qui rejoint les préoccupations de Brahms lui-même qui, dans les leçons qu'il donnait à Gustav Jenner, recommendait d'apporter le plus grand soin dans le choix et la disposition des cadences). Dans cette perspective, la cadence joue

${ }^{20}$ Le thème principal de l'op. 60 présente également un processus d'expansion de la longueur des segments similaire à celui du thème de la Symphonie $n^{\circ} 1$ en do mineur, op. 68 , et un patron expressif en trois temps similaire à celui de l'op. 34 : les deux œuvres débutent par une section dépouillée (mes. 1-4 pour le quintette, avec une simple ligne non harmonisée; et mes. 1-27 pour le quatuor). Puis une section produisant une intensification mène à une section plus dramatique (les sections d'intensification et dramatique se produisent respectivement aux mes. 5-12 et 13-23 pour le quintette, et aux mes. 27-31 et 32-42 pour le quatuor). Ce patron semble trouver son origine chez les classiques et est illustré par exemple dans les thèmes principaux des mouvements initiaux de la Sonate pour violon en mi mineur, K. 304, de Mozart et de la Sonate pour piano en fa mineur, op. 57 ( Appassionata »), de Beethoven. Il est associé à d'autres patrons formels chez Brahms, comme dans le thème principal du Quatuor avec piano en sol mineur, op. 25, le thème initial de l'Ouverture tragique, op. 81 (deux exemples qui exploitent comme l'op. 34 l'opposition entre une section initiale monodique et son retour harmonisé). 
un rôle de jonction important entre la forme et l'organisation tonale. Du point de vue formel, elle articule la fin d'un groupe et exprime une fonction conclusive et, du point de vue harmonique, elle renseigne sur l'organisation tonale du groupement. La nature de cette information est déterminée par les conventions qui lient la cadence à l'organisation de la tonalité sous-jacente, et elle varie selon que l'organisation cadentielle se produit dans un contexte de monotonalité, de complexe de double tonique ou de tonalité progressive. La monotonalité désigne une organisation tonale qui débute et se termine dans la même tonalité et considère les modulations comme des expansions des degrés harmoniques de la tonalité principale. Cette conception de la tonalité est associée entre autres aux travaux de Schönberg et de Schenker. Le complexe de double tonique s'applique à une œuvre ou à la section d'une œuvre qui peut s'interpréter simultanément en fonction de deux tonalités complémentaires, et la tonalité progressive s'applique aux œuvres qui débutent et se terminent dans deux tonalités différentes.

Les structures hiérarchiques du groupement formel et de l'organisation tonale font l'objet d'une stricte coordination dans la monotonalité classique. Dans le cas de la forme sonate, par exemple, la structure hiérarchique du groupement formel s'exprime au niveau supérieur par la division de la forme générale en trois sections - l'exposition, le développement et la récapitulation -, auxquelles peuvent s'ajouter une introduction lente et une coda. Chaque section se subdivise à son tour : l'exposition est constituée de trois parties - un thème principal, une transition et un thème subordonné - qui se ramifient encore.

L'organisation tonale repose également sur une organisation hiérarchique. Les modulations sont coordonnées d'après un plan d'ensemble où la modulation du ton principal au ton subordonné est résolue avec le retour du ton principal. Les différentes possibilités de tons subordonnés sont $\mathrm{V}$ en majeur et III et $\mathrm{v}$ en mineur (l'exploration d'autres tonalités chez Beethoven constitue un cas particulier). D'autres modulations peuvent se produire à l'intérieur des sections thématiques ou dans le développement. Du point de vue hiérarchique, le ton subordonné est considéré au niveau supérieur comme un satellite du ton principal ou comme un degré intermédiaire dans le mouvement de la tonique vers la dominante qui termine le développement. À un niveau inférieur, les autres modulations sont considérées comme des satellites du ton principal, du ton subordonné ou du mouvement de l'un à l'autre. Dans les sections thématiques, les différents tons satellites entretiennent les mêmes rapports avec le ton de référence (principal ou subordonné) que les différentes possibilités de tonalités subordonnées par rapport au ton principal : $\mathrm{V}$ pour une tonalité majeure, III ou v pour une tonalité mineure. Par exemple, le thème initial du finale du Quatuor en do mineur, op. 18, $\mathrm{n}^{\circ} 4$, de Beethoven (mes. 1-16) produit une modulation intérieure à la dominante mineure (mes. 8), ou le thème du mouvement lent du Concerto pour piano $\mathrm{n}^{\circ} 18$ en la majeur, K. 456, de Mozart (mes. 1-21), écrit dans un ton mineur, produit à la mes. 8 une modulation intérieure au ton relatif. On retrouve parfois des modulations à des tons inhabituels, comme dans les refrains des derniers mouvements des Symphonies $\mathrm{n}^{\text {os }} 88$ et 102 de Haydn (deux cadences au ton de iii d'une tonalité majeure, 
respectivement une cadence authentique parfaite et une demi-cadence), mais elles sont rares.

La monotonalité classique se fonde sur une relation étroite entre la modulation et la cadence. Une cadence confirme une modulation et, inversement, une tonalité satellite n'est reconnue que si elle est établie par une cadence. Dans la forme sonate, tous les groupes thématiques sont confirmés par une cadence et tous les groupes de thèmes subordonnés sont confirmés par une cadence authentique. Cela signifie donc que la cadence peut jouer deux rôles dans une section thématique :

1. à la fin du thème, elle confirme le ton de référence pour l'ensemble d'un groupe formel;

2. à l'intérieur d'un thème, la cadence confirme soit le ton de référence, soit le chemin parcouru à l'intérieur du groupe formel (comme dans la cadence authentique parfaite à la fin de la section A d'une petite forme ternaire sur les degrés $V$ pour les deux modes, ou III et $v$ en mineur).

Certains cas exceptionnels brisent la relation entre cadence et tonalité. Les sections thématiques situées à la fin de trios (de scherzo ou de menuet) ou de couplets de rondo ne sont pas toujours confirmées par des cadences. L'absence de cadence produit un effet spécial, et c'est là une manière de conférer à la section une certaine ouverture et de favoriser l'enchaînement avec la section suivante. Cela exprime également la subordination de la tonalité de la section ouverte par rapport au ton principal.

La plupart du temps, la musique de Brahms remplace la relation strictement réciproque entre ton et cadence par une relation plus souple entre tonalité et geste cadentiel et, si elle préserve généralement la monotonalité, elle manifeste souvent un élargissement de l'espace tonal. L'organisation harmonique des œuvres monotonales de Brahms exploite des tonalités plus éloignées que celles des classiques, à la fois sur le plan de la forme générale dans le choix du ton subordonné, et sur le plan thématique dans les cadences internes sur des tons satellites aux tons principaux et subordonnés.

L'élargissement de l'espace tonal débute déjà avec Beethoven. En ce qui concerne la forme générale, Beethoven écrit des thèmes subordonnés dans les tons de la médiante en majeur, et de la sous-médiante dans les deux modes. Oster décrit la fonction qu'assure chaque degré dans la structure harmonique générale et montre que plusieurs des nouvelles possibilités explorées par Beethoven ont été reprises par les compositeurs romantiques, dont Brahms (il mentionne l'utilisation de vi dans l'arpégiation $\mathrm{i}-\mathrm{vi}-\mathrm{iv}-\mathrm{V}$ dans le Quintette avec piano, op. 34, et III dans l'arpégiation I-III-V-I dans les premier et dernier mouvements du Quintette à cordes en fa majeur, op. 88, et dans la Sonate pour violoncelle $\mathrm{n}^{0} 2$ en fa majeur, op. 99) ${ }^{21}$. Rosen mentionne aussi un trait particulier

${ }^{21}$ Ernst Oster, dans les annotations à sa traduction d'Heinrich Schenker, Free Composition Vol. III of New Musical Theories and Fantasies (New York et Londres : Longman, 1979), 139. 
à Brahms dans le choix des tons subordonnés : l'accent mis sur la dominante mineure (par exemple, dans les premiers mouvements des Concertos pour violon en ré majeur, op. 77 , et pour piano $n^{\circ} 2$ en si bémol majeur, op. 83$)^{22}$. Même si Rosen reconnaît une source probable de ce procédé dans la mixture modale des tons subordonnés (III-iii) dans la Sonate pour piano, op. 57 («Appassionata »), de Beethoven, il affirme que c'est seulement chez Brahms que le procédé atteint une telle ampleur.

L'utilisation de l' «exposition à trois tonalités » constitue une autre manifestation à grande échelle de l'élargissement des relations tonales au sein de la monotonalité. Traditionnellement, l'exposition classique est fondée sur une dramatisation de la modulation au ton subordonné, et présente donc un conflit entre les tons principal et subordonné23. Ce type d'organisation tonale est remis en cause par l'exposition à trois tonalités, qui, comme son nom l'indique, établit trois tons dans le courant de l'exposition plutôt que deux. Les premier et troisième tons assument généralement les fonctions de ton principal et de ton subordonné, et la seconde tonalité joue un rôle de relais entre les deux. Le matériau associé à la seconde tonalité manifeste souvent une ambiguitté formelle : il est généralement introduit comme s'il s'agissait du thème subordonné, mais la suite montre rétrospectivement qu'il ne marque qu'une étape avant la véritable arrivée du ton subordonné24.

Chez les classiques, les expositions à trois tonalités sont relativement rares, et la seconde tonalité est très faiblement établie : elle ressemble à un embellissement, une tonicisation appuyée survenant au milieu du parcours tonal menant au ton subordonné ${ }^{25}$. Chez Brahms et les romantiques, la seconde tonalité est établie beaucoup plus fermement, et elle est souvent confirmée par des cadences.

Les études théoriques sur les expositions à trois tonalités se concentrent généralement sur l'organisation harmonique et tendent, sauf exception, à négliger la dimension formelle. Celle-ci joue pourtant un rôle important dans la réception et l'impact de l'exposition à trois tonalités. Habituellement, le matériau du second ton exprime une structure formelle thématique assez développée et débute par une fonction d'initiation claire. La tonalité transitoire est parfois même préparée par une station sur la dominante, comme dans le

22 Charles Rosen, « Brahms the Subversive », dans Brahms Studies : Analytical and Historical Perspectives, édité par George S. Bozarth (Oxford : Clarendon Press, 1990), 108.

${ }^{23}$ Roger Graybill appelle «modèle bipolaire » (bipolarity model) ce modèle souvent associé aux travaux de Rosen; voir «Brahms's Three-Key Expositions : Their Place Within the Classical Tradition» (thèse de doctorat, Yale University, 1983), 14-15.

${ }^{24}$ Graybill offre dans certains cas une explication plus complexe, qui fait appel à un phénomène de circularité entre la fin du groupe subordonné et la reprise de l'exposition, comme dans le mouvement initial de la Sonate pour violoncelle $\mathrm{n}^{\circ} 2$ en fa majeur, op. 99, voir « Brahms's ThreeKey Expositions », 277-319, et « Harmonic Circularity in Brahms's F Major Cello Sonata : An Alternative to Schenker's Reading in "Free Composition" ", Music Theory Spectrum 10 (1988) : 43-55.

${ }^{25}$ C'est ce que montre Rosen au sujet du premier mouvement du Concerto pour piano ${ }^{\circ} 5$ («Empereur ») de Beethoven dans Sonata Forms, éd. rév. (New York :W.W.Norton, 1988), 249-56, ou Caplin au sujet de l'Ouverture Coriolan de Beethoven dans Classical Form, 119-21. 
premier mouvement de la Sonate pour violoncelle et piano $\mathrm{n}^{\circ} 2$ en fa majeur, op. 99 , de Brahms (la brève station sur la dominante précédant la structure thématique associée au deuxième ton se produit aux mes.30-32). Dans le dernier mouvement de la Sonate pour violon $\mathrm{n}^{\circ} 3$ en ré mineur, op. 108 , le ton de la station sur la dominante contredit celui du ton intermédiaire et annonce en fait le véritable ton subordonné ${ }^{26}$.

Les structures à retour jouent un rôle particulièrement important dans l'articulation des expositions à trois tonalités chez Brahms. Les modulations du second au troisième ton sont souvent produites par une période modulante (comme dans les premiers mouvements de la Sonate pour violoncelle $\mathrm{n}^{\circ} 2$, op. 99, du Trio pour clarinette (ou alto), violon et piano en la mineur, op. 114, de la Sonate pour clarinette en fa mineur, op. $120, \mathrm{n}^{\circ} 1$, et pour la forme rondo, dans les finales du Trio avec piano $\mathrm{n}^{\circ} 2$, op. 87, de la Sonate pour violoncelle $\mathrm{n}^{\circ} 2$, et de la Sonate pour violon $\mathrm{n}^{\circ} 3$ ). L'antécédent est généralement relativement bref et conventionnel, et il débute par une fonction d'initiation claire qui semble marquer le début du thème subordonné. Le conséquent est plus développé, et assure le rôle de transition entre le ton intermédiaire et le ton subordonné.

Par exemple, l'exposition du premier mouvement du Trio, op. 114, établit successivement les tons de $l a$ mineur, de $d o$ majeur et de mi mineur. Le premier ton est articulé par le thème principal (mes.1-33), et les deux derniers tons sont organisés en fonction d'une période modulante (mes. 44-67). L'organisation formelle contribue à établir le second ton avec une fermeté inconnue des expositions à trois tonalités classiques : il est d'abord exprimé par deux gestes conclusifs, la cadence parfaite qui termine la transition mes. 44 (l'utilisation de ce type de cadence à ce point est un procédé inhabituel), et l'arrivée de la dominante qui conclut l'antécédent. Le ton de do est également articulé par la fonction d'initiation de l'antécédent et du conséquent. L'expression de la fonction d'initiation est définie par l'établissement des références, dont celle de la tonalité. Ainsi, la prolongation de la tonique au début de l'antécédent et du conséquent sert à la fois à caractériser la fonction d'initiation et à projeter la seconde tonalité. Si l'arrivée du troisième ton (soit le ton subordonné réel) n'est articulée par aucune fonction d'initiation, il est établi avec force par la série de gestes cadentiels, d'abord évités, puis finalement aboutis. L'utilisation de la période pour articuler les tons finaux d'une exposition à trois tonalités est un procédé fréquent chez Brahms, bien qu'il ne lui soit pas exclusif. Un autre exemple se produit dans le premier mouvement de la Sonate pour violoncelle en sol mineur, op. 65, de Chopin. Néanmoins, les romantiques lui préfèrent généralement d'autres techniques ${ }^{27}$.

${ }^{26}$ La divergence entre le ton suggéré par la station sur la dominante qui termine la transition et celui du thème subordonné est un procédé souvent utilisé indépendamment de l'exposition à trois tonalités chez les romantiques : on la trouve par exemple dans le premier mouvement de la Sonate pour violoncelle $\mathrm{n}^{\circ} 1$ en si bémol majeur, op. 45 , de Mendelssohn. L'utilisation de ce procédé chez Brahms rappelle plutôt l'exemple qu'on trouve dans le finale de la Sonate pour violon $n^{\circ} 2$ en ré mineur op. 121, de Schumann, un trait qui vient s'ajouter à plusieurs autres points communs entre les deux œuvres (le ton de ré mineur, un fugato sur un thème présentant un déplacement rythmique d'une croche, etc.) et qui suggère l'influence de la pièce de Schumann sur celle de Brahms.

${ }^{27}$ Un autre type d'organisation formelle associé aux expositions à trois tonalités consiste à 
Brahms exploite souvent les structures à retour dans d'autres cas de tonalités illusoires analogues à l'exposition à trois tonalités, soit dans des passages qui créent l'illusion de l'arrivée formelle d'une section thématique sur une tonalité qui se révèle rétrospectivement être la mauvaise. Un cas fréquemment cité dans la littérature théorique est l'utilisation de la petite forme ternaire dans la fausse récapitulation ${ }^{28}$. La majorité des fausses récapitulations des œuvres instrumentales de Brahms surviennent dans les mouvements lents dont elles exploitent les particularités structurelles typiques ${ }^{29}$. Chez ce compositeur, les mouvements lents sont habituellement écrits dans la grande forme ternaire (ou un dérivé) et les sections $\mathrm{A}$ et $\mathrm{B}$ adoptent la petite forme ternaire. La fausse récapitulation présente la section $\mathrm{A}$ de la petite forme ternaire dans la mauvaise tonalité, la section médiane contrastante qui débute dans la mauvaise tonalité joue le rôle de retransition en modulant au ton principal, et la véritable récapitulation commence avec la section $\mathrm{A}^{\prime}$, entièrement présentée à la tonique.

L'utilisation combinée de la fausse récapitulation et de la petite forme ternaire typique de la section A permet à Brahms de faire d'une pierre deux coups. En effet, cela lui permet (1) de commencer à la fois la fausse récapitulation et la véritable récapitulation par l'idée de base du thème; et (2) d'obtenir une présentation du matériau thématique complet, sans redondance.

Certaines périodes produisent une variante du même procédé. L'antécédent débute dans la mauvaise tonalité, mais il produit une modulation au ton initial de manière à ce que le conséquent soit entièrement présenté dans la tonique. Cette variante se produit dans la récapitulation du thème subordonné du premier mouvement de la Sonate pour alto en mi bémol majeur, op. 120, $\mathrm{n}^{\circ} 2$. La prédilection que manifeste la musique de Brahms pour l'utilisation des structures à retour dans les expositions à trois tonalités et les fausses récapitulations témoigne à la fois de son importance dans l'écriture de ce compositeur et de la faculté d'adaptation de ce type formel à l'égard des nouveaux problèmes formels et musicaux propres au XIX $\mathrm{X}^{\mathrm{e}}$ siècle.

Un autre témoignage de l'élargissement de l'espace tonal chez les romantiques se manifeste sur le plan thématique par l'utilisation de cadences intérieures sur des degrés inhabituels, différents de V en majeur, et de III et de $\mathrm{v}$ en mineur. Une fois de plus, Beethoven fait figure de précurseur. Schönberg

articuler la seconde tonalité par une section thématique et introduire la troisième tonalité à l'aide d'une section modulante. Ce procédé est particulièrement fréquent chez Schubert, qui l'utilise par exemple dans les premiers mouvements de la Sonatine pour violon et piano $\mathrm{n}^{\circ} 3$ en sol mineur, $\mathrm{D}$. 408, et du Quatuor à cordes en ré mineur, D. 810 («Der Tod und das Mädchen »), dans le Quartett$S a t z$, D. 703 , les premier et dernier mouvements de la Symphonie $n^{\circ} 2$, D. 125 , et le finale de la Symphonie $\mathrm{n}^{\circ}$ 3, D. 300.

${ }^{28}$ Elaine Sisman, « Brahms's Slow Movements : Reinventing the "Closed" Forms », dans Brahms Studies: Analytical and Historical Perspectives, 79-104; 86-95; Peter Smith, « Formal Ambiguity and Large-Scale Tonal Structure in Brahms's Sonata-Form Recapitulations » (thèse de doctorat, Yale University, 1992), 152-57 et passim.

${ }^{29}$ Deuxièmes mouvements du Quatuor à cordes $n^{\circ} 2$ en la mineur, op. $51, n^{\circ} 2$; du Quatuor à cordes $n^{\circ} 3$ en si bémol majeur, op. 67; du Quintette à cordes $n^{\circ} 1$ en fa majeur, op. 88; de la Sonate pour violon $\mathrm{n}^{\circ} 2$ en la majeur, op. 100 . Troisièmes mouvements de la Sérénade $\mathrm{n}^{\circ} 1$ en ré majeur, op. 11; du Quatuor avec piano ${ }^{\circ} 2$ en sol mineur, op. 25; du Concerto pour piano $n^{\circ} 2$ en si bémol majeur, op. 83, etc. 
cite l'exemple de l'Allegretto du Quatuor à cordes en mi mineur, op. 59, $\mathrm{n}^{\prime \prime} 2^{30}$. Le thème initial est une période dont le conséquent se termine à la mes: 8 par une cadence authentique parfaite dans le ton de bVII. Schönberg mentionne aussi le finale du Quatuor à cordes en la mineur, op. 132, dont le thème principal adopte une coupe de petite forme binaire, et dont la première partie se termine par une demi-cadence dans le ton de III (mes. 10), soit, dans les faits, une autre articulation sur bVII.

Brahms explore trois tonalités non traditionnelles dans les cadences internes des structures à retour, le III\#en majeur, le VI diatonique (bVI en mineur ou vi en majeur), et le bVII en mineur. Le thème initial du finale du Quintette avec piano en fa mineur, op. 34, servira à illustrer l'utilisation d'une cadence interne au ton dans le ton de $b \mathrm{VI}^{31}$. Ce passage adopte une organisation particulière, inusitée chez les classiques, mais relativement fréquente chez Brahms, que nous qualifierons de «structure bipartite équilibrée ». Il s'agit d'une période dont l'antécédent et le conséquent sont suivis respectivement d'une station sur la dominante et d'une section conclusive, et dans laquelle les deux sections postcadentielles sont fondées sur le même matériau. Ainsi, un effet de rime double est créé par la reprise à la fois du matériau de l'antécédent au début du conséquent, et par celui de la station sur la dominante dans la section conclusive $^{32}$. Ici, l'antécédent des mes. 42-53 est suivi d'une station sur la dominante aux mes. 54-57, et le conséquent des mes. 58-71 est suivi d'une section conclusive aux mes. 72-79. Le début de la section conclusive reprend le même matériau que la station sur la dominante. L'antécédent et le conséquent se terminent respectivement par une cadence rompue dans le ton de la dominante mineure, réinterprétée comme une arrivée de la dominante à la mes. 53, et par une cadence authentique parfaite dans le ton principal (avec une tierce picarde) à la mes. 71 . Ces cadences ne sont pas atteintes d'un seul coup, mais elles sont à chaque fois précédées d'une ou deux tentatives cadentielles : l'antécédent présente une demicadence dans le ton de la dominante mineure à la mes. 49 , et le conséquent produit une cadence imparfaite dans le ton de bVI à la mes. 65 et une cadence rompue dans le ton initial à la mes. 69. Les cadences dans le ton de $v$ et bVI sont

\footnotetext{
${ }^{30}$ Arnold Schönberg, Structural Functions of Harmony (New York : W. W. Norton, 1969 [éd. orig., 1954]), 74 .

${ }^{31}$ On retrouve des exemples de cadence interne sur d'autres degrés dans les œuvres suivantes. Le degré III \#dans un ton majeur est utilisé dans le thème principal du Quintette à cordes en fa, op. 88, dans le thème du finale du Quatuor à cordes $n^{\circ} 3$ en si bémol majeur, op. 67 (un thème et variations), et dans le thème subordonné du premier mouvement du Quintette à cordes en fa majeur, op. 88. La cadence sur bVII est illustrée dans le premier couplet du finale de la Sonate pour violon $\mathrm{n}^{\circ} 1$ en sol majeur, op. 78, et dans le second mouvement de la Sonate pour clarinette en mi bémol majeur, op. 120.

${ }^{32}$ Quelques exemples de ce type de structure se produisent dans les thèmes initiaux du troisième mouvement du Trio avec clarinette en la mineur, op. 114; dans les quatrièmes mouvements du Quatuor à cordes $\mathrm{n}^{\circ} 2$ en la mineur, op. $51, \mathrm{n}^{\circ} 2$, et du Trio avec clarinette en la mineur, op. 114; et dans les thèmes subordonnés des premiers mouvements de la Sonate pour piano $\mathrm{n}^{\circ} 1$ en do majeur, op. 1, du Trio avec piano $\mathrm{n}^{\circ} 1$ en si majeur, op. 8 (dans la version originale), et du Quintette avec piano en fa mineur, op. 34. Il existe également une variante de forme ternaire, dans lequel le matériau de $B$ est repris dans la codetta, comme dans le thème initial du deuxième mouvement du Quatuor à cordes $n^{\circ} 2$ en la mineur, op. $51, n^{\circ} 2$.
} 
inhabituelles à cause de leur éloignement de la tonique, et elles sont suivies d'extensions qui amènent les cadences finales des deux membres. La cadence sur $b$ VI a pour fonction d'approcher la dominante dans la progression I-bVI-V. La dominante est abordée une première fois à la mes. 69 , mais une cadence rompue ramène le bVI. La véritable arrivée de $\mathrm{V}$ se situe donc à la mes. 71 et se résout sur $\mathrm{i}$.

D'autres thèmes exploitent la modulation interne à VI dans d'autres fonctions harmoniques. Le refrain du finale du Concerto pour violon en ré majeur, op. 77, est construit sur une petite forme ternaire dont la section A se termine par une cadence authentique parfaite sur vi. Lorsque la cadence s'enchaîne à la répétition de A (mes. 8-9), elle assure un rôle harmonique de I-vi-I, et lorsqu'elle s'enchaîne à la section B (16-17), elle produit la progression I-vi-III\#-V.

Parallèlement à la monotonalité, les romantiques explorent des organisations tonales différentes : la tonalité progressive et le complexe de double tonique. Ces types d'organisation entraînent une redéfinition de la signification de l'organisation cadentielle. Le complexe de double tonique s'applique à une œuvre ou à la section d'une œuvre qui peut s'interpréter simultanément en fonction de deux tonalités complémentaires (généralement en rapport de tierce). L'organisation tonale du passage ne peut s'analyser en fonction d'une tonalité unique, ni par la modulation d'un ton à un autre, mais seulement par la combinaison de deux tonalités ${ }^{33}$. Schubert est probablement un des premiers compositeurs à utiliser ce type d'écriture dans la musique instrumentale de tradition sérieuse (dans le thème subordonné du premier mouvement de l'Octuor en fa majeur, D. 803, par exemple). Brahms a utilisé à quelques reprises le complexe de double tonique (complexe de sol majeur et mi mineur dans le premier couplet du finale de la Symphonie $\mathrm{n}^{\circ} 1$ en do mineur, op. 68; complexe de sol majeur et si mineur dans le thème subordonné de la Sonate pour violoncelle $\mathrm{n}^{\circ} 1$ en mi mineur, op. 38).

Un passage du finale du Trio avec piano $\mathrm{n}^{\circ} 3$ en do mineur, op. 101, servira d'exemple pour illustrer l'utilisation d'une structure à retour dans le contexte d'un complexe de double tonique (exemple 6). Le groupe subordonné couvre les mes. 35-84; si le deuxième thème subordonné qui va de la cadence de la mes. 66 à la fin du groupe exprime clairement le ton de sol mineur (dominante mineure du ton principal), le premier thème subordonné aux mes. 35-66 entretient une ambiguïté constante entre les tons de sol mineur et de $s i$ bémol majeur. Ce passage se divise en deux. La première partie comporte deux phrases (mes. 35-39, 41-48), dont la seconde constitue une répétition de la première phrase avec une extension, et dont le début est anticipé par un accord à la mes. 40. La seconde partie est dérivée d'une structure périodique, et ses deux membres (mes. 50-57,58-66) présentent tous les deux une structure similaire à une proposition (avec des présentations aux mes. 50-53 et 58-61 et des continuations aux mes. 54-57 et 62-66).

${ }^{33}$ Ce type d'organisation est décrit dans les travaux suivants : Robert Bailey, édit., Richard Wagner: Prelude and Transfiguration from "Tristan and Isolde", Norton Critical Scores (New York : W. W. Norton, 1985); Christopher Lewis, «Mirrors and Metaphors : On Schoenberg and Nineteenth-Century Tonality », dans Music at the Turn of the Century : A 19th-Century Music Reader, édité par Joseph Kerman (Berkeley et Los Angeles : University of California Press, 1990), 15-31. 
L'ambiguïté tonale exploite un procédé dans lequel un élément stable présenté dans deux contextes différents suggère alternativement une des deux interprétations tonales. Ce procédé est réalisé de deux manières différentes. Dans la première, une progression à la tonalité ambiguë reçoit une terminaison qui suggère une tonalité, et la progression est répétée avec une extension qui suggère l'autre tonalité. L'extension entraîne un effet de réinterprétation rétroactive et prouve que le même passage peut s'interpréter dans les deux tons. Dans l'autre exemple, un contour mélodique invariant est harmonisé de deux façons différentes, selon chaque tonalité. Ces deux techniques sont illustrées dans la première partie.

La phrase initiale débute dans un contexte harmonique ambigu (mes. 35) et se termine par une cadence authentique parfaite dans le ton de si bémol (mes. 39), un geste conclusif qui tend à confirmer la prédominance de cette tonalité. Mais la phrase est répétée aux mes. 41-45 avec une extension produisant une cadence parfaite sur ré (mes. 48). Le ton de sol affirmé dans les mesures suivantes suggère la réinterprétation de la cadence sur ré comme une demi-cadence dans le ton de sol mineur, et, si on pousse l'interprétation rétroactive plus loin, définit la cadence des mes. 39 et 45 sur si bémol comme une articulation du relatif (III). De plus, la progression cadentielle sur ré (mes. 46-48) maintient le même contour mélodique (avec quelques modifications d'altérations) que la progression cadentielle sur si bémol (mes. 43-45), tel qu'illustré par des crochets à l'exemple 6 . Ce contour, basé sur les notes sol-fa-ré, apparaît dès la fin du thème principal (mes. 31-33) et revient plus loin dans le second thème subordonné (mes. 71-72).

La seconde partie du thème présente la structure plus familière de période, qui, loin de lever les doutes sur l'organisation tonale, en renforce l'ambiguïté (voir les mes. 50-66 à l'exemple 6 et le schéma de la figure 4). Le début de l'antécédent établit habituellement le ton de référence. Ici, la ligne monodique par laquelle commence le membre n'exprime pas la tonique, mais la succession d'accords des mes. 52-54 semble établir le ton de sol mineur par la progression iv $-V^{9}-\mathrm{i}$. La cadence parfaite sur $f a$ à la fin de l'antécédent et le retour sur $s i$ bémol renversent cette première intuition et suggèrent fortement une structure en $s i$ bémol dont l'antécédent débute sur vi et se termine par une cadence réinterprétée (PAC V $\Rightarrow \mathrm{HC}$ ), suivie du retour habituel sur I. Le changement de tonalité au début du conséquent exploite le procédé d'invariance du contour mélodique par rapport à l'antécédent, si on excepte l'altération du fa bécarre (comme le montre la comparaison des segments indiqués par des crochets aux mes. 52-53 et 60-61 de l'exemple 6). Le premier geste cadentiel du conséquent, la cadence évitée de la mes. 65, constitue une transposition partielle de la cadence de l'antécédent et aurait pu clore le thème dans le ton de si bémol. Une extension combinée avec l'invariance du contour mélodique mène à une cadence sur sol. L'extension oblige à réinterpréter en sol mineur une phrase qui aurait aussi bien pu s'analyser en si bémol. Le second thème subordonné établit le ton de $s o l$ sans ambiguité, mais l'extension fait une dernière allusion discrète au ton de si bémol : les accords VI-iv-VII ${ }^{7}$ aux mes. 70-71 renvoient aux accords IV-ii- $\mathrm{V}^{7}$ en si bémol (ces accords supportent le motif sol-fa-réà la mélodie). 
Exemple 6 : Brahms, Trio avec piano $n^{\circ} 3$ en do mineur : $4^{\mathrm{e}}$ mouvement, mes. 31-84
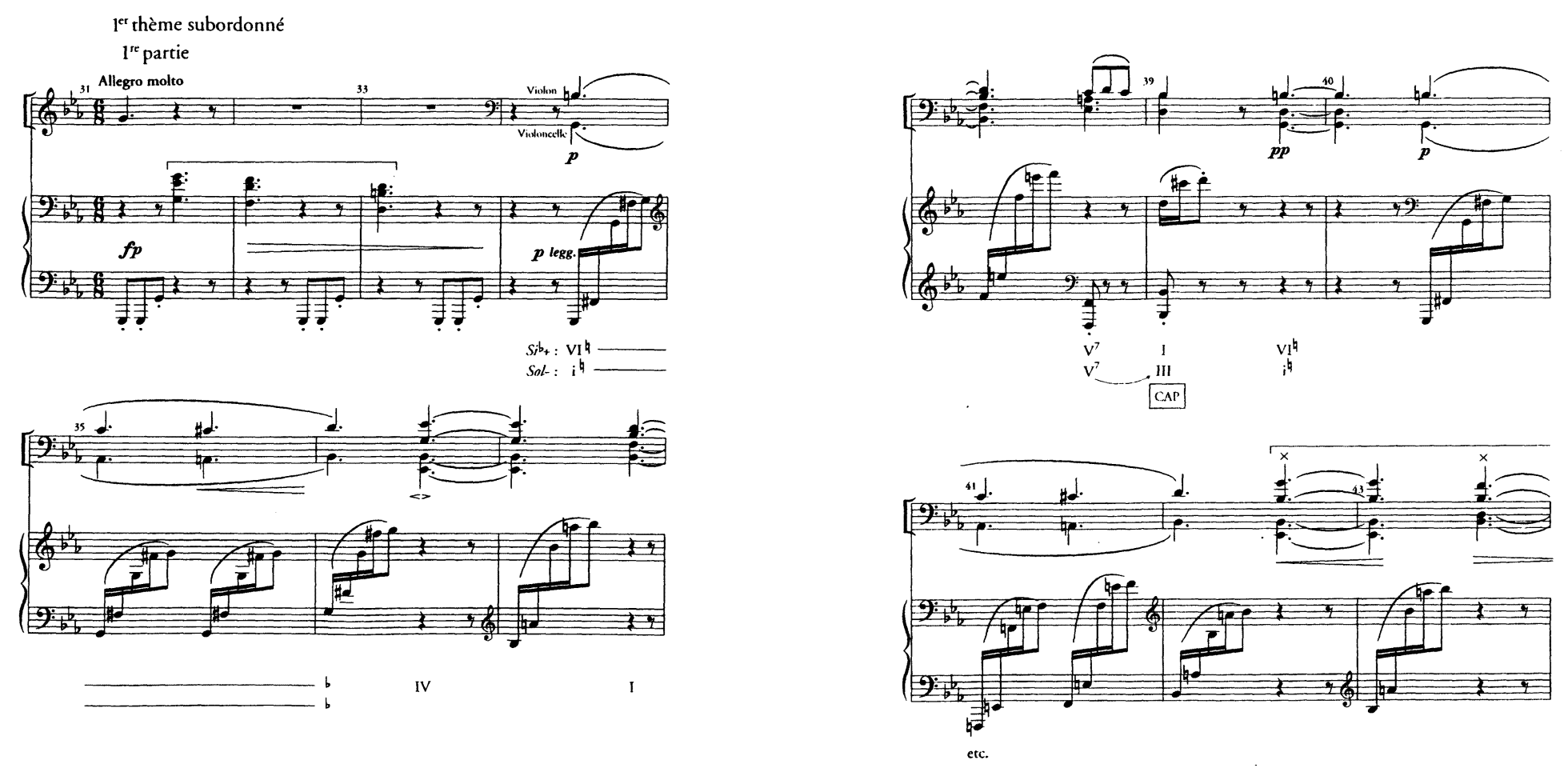

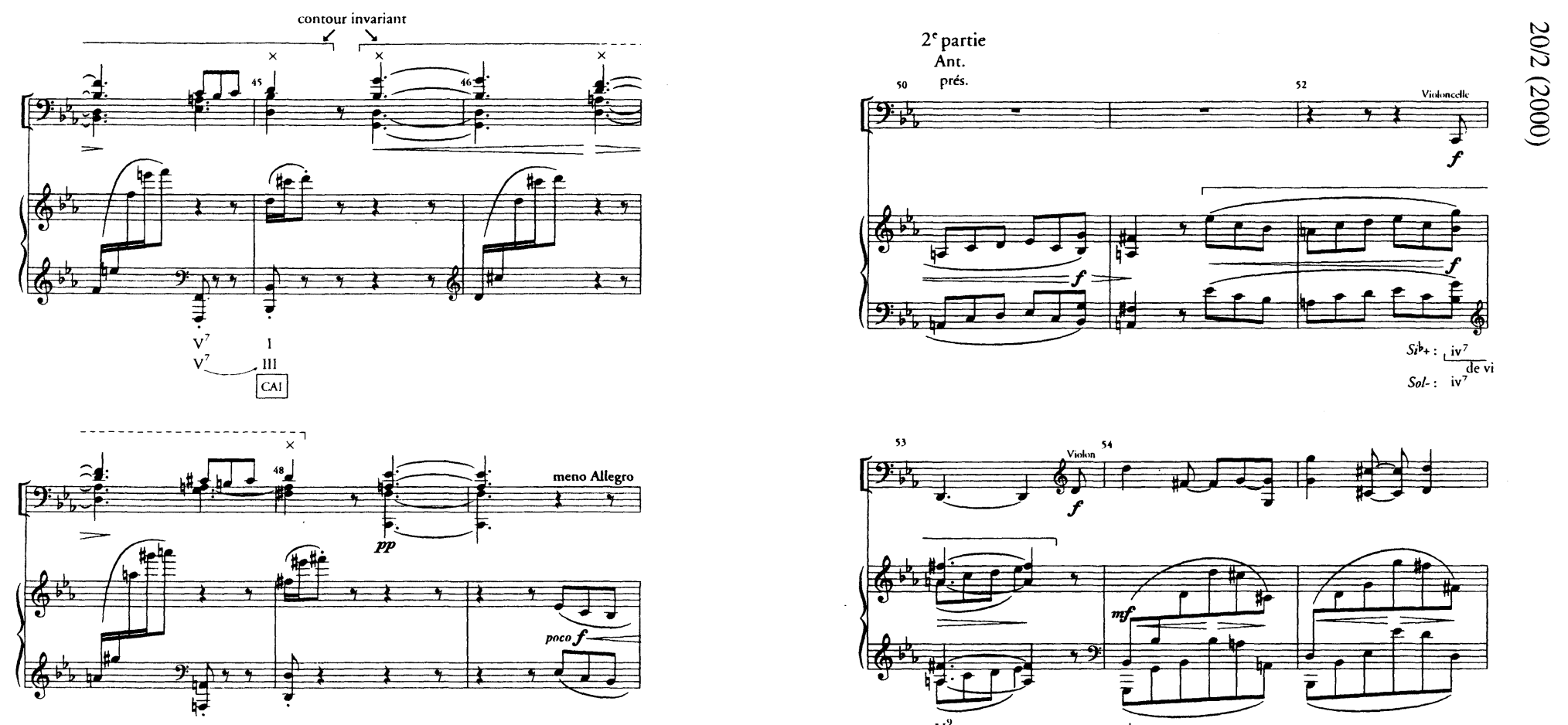

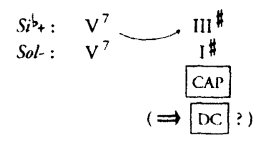

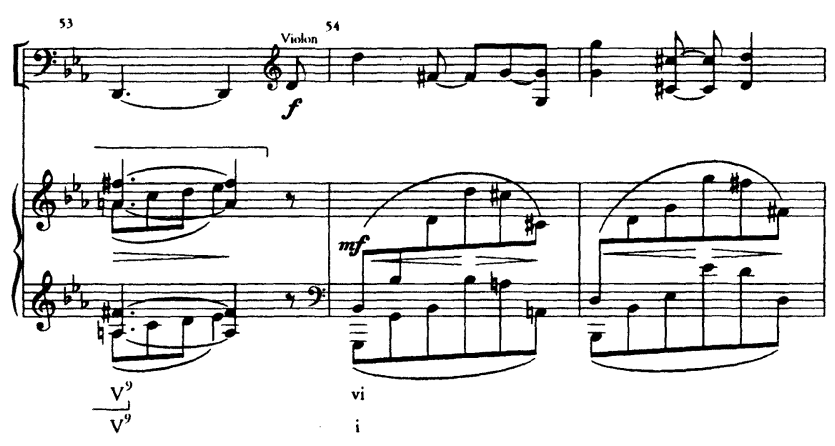



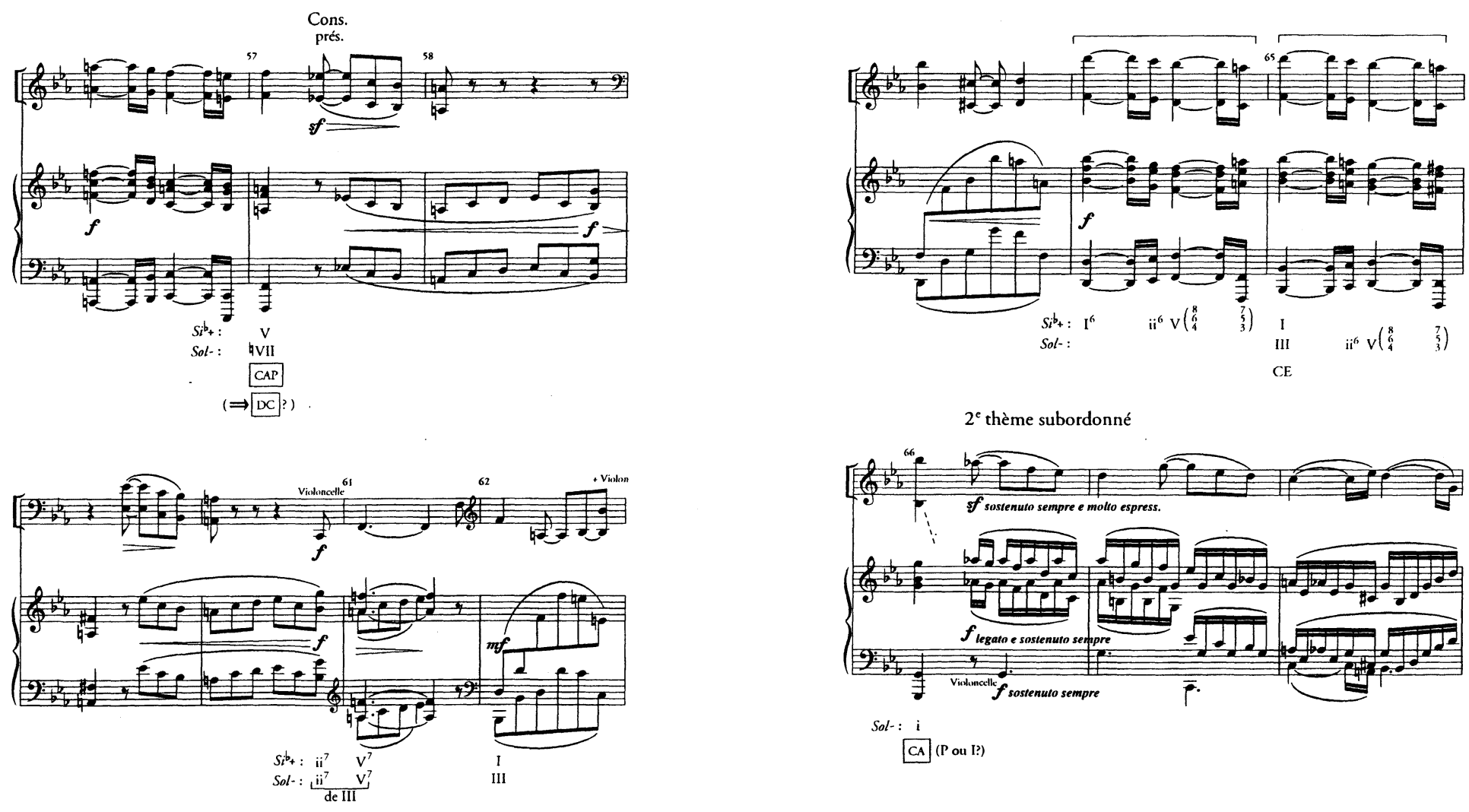

Sol : : i
CA (P ou I?) 

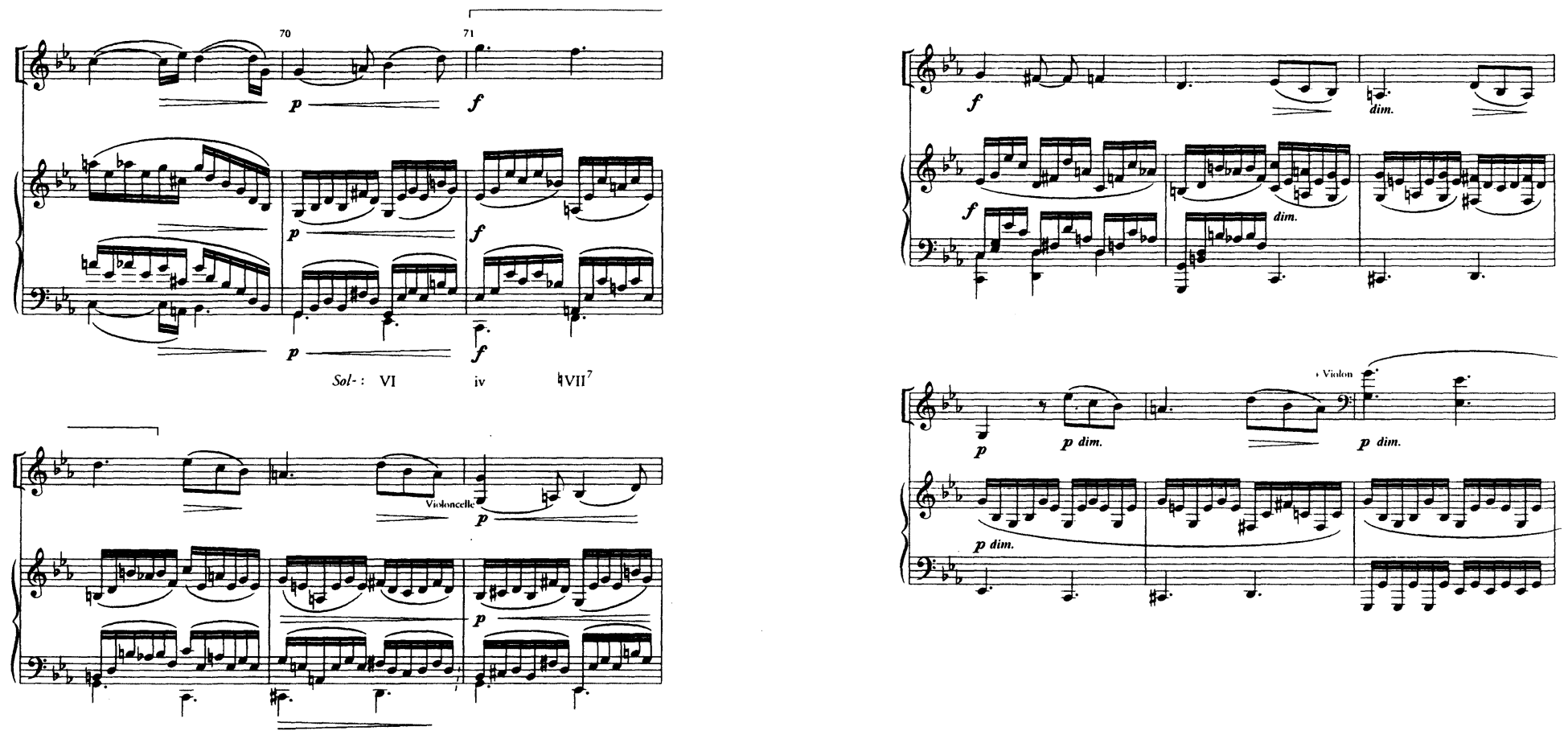


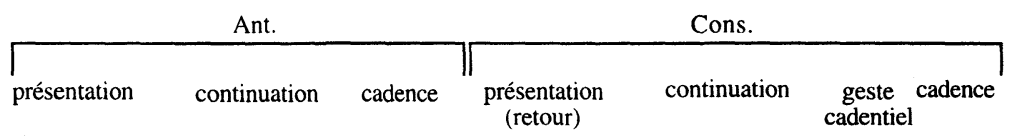

\begin{tabular}{|c|c|c|c|c|c|c|c|}
\hline Si bémol + & iv 7 & $\mathrm{~V}^{9}$ & vi & $C A P V \Rightarrow D C$ & ii $^{7} \quad V^{7}$ & I & CE I CA vi \\
\hline Sol - : & iv 7 & $\mathrm{~V}^{9}$ & $\mathrm{i}$ & CAP VII & $\frac{\mathrm{ii}^{7} \mathrm{~V}^{7}}{\text { III }}$ & III & CE III CA i \\
\hline Mesure : & 50 & & 54 & 57 & 58 & 62 & 65 \\
\hline
\end{tabular}

Figure 4 : Brahms, Trio avec piano $\mathrm{n}^{\circ} 3$ en do mineur, op. $101: 4^{\mathrm{e}}$ mouvement, mes. 50-66

Les cadences jouent une fonction différente dans la monotonalité et dans le complexe de double tonique. Dans le premier cas, la cadence apporte soit la confirmation du ton de référence, soit la confirmation du chemin harmonique parcouru. Ces deux fonctions sont impossibles dans le complexe de double tonique : l'ambiguïté constante entre deux pôles empêche l'établissement d'un ton de référence, et l'évaluation du chemin parcouru est impossible faute de points de repère. Les cadences internes ont plutôt pour rôle d'articuler les degrés caractéristiques des deux tons du complexe et de contribuer à l'ambiguïté tonale jusqu'à la clarification de la cadence finale. L'utilisation de la période dans le complexe de double tonique montre la faculté d'adaptation de la structure à retour aux nouvelles techniques tonales développées au XIX $\mathrm{X}^{\mathrm{e}}$ siècle.

La musique romantique explore également un autre type d'organisation tonale, appelée tonalité progressive ${ }^{34}$. La tonalité progressive s'applique aux œuvres qui débutent et se terminent dans deux tonalités différentes. L'utilisation de ce type d'organisation est particulièrement problématique dans la musique instrumentale, car l'élimination d'une référence tonale unique rend impossible le processus de résolution formelle et tonale qui sous-tend les formes instrumentales. L'œuvre de Brahms présente peu d'exemples de tonalité progressive, et ils se produisent plutôt dans les genres différents de la sonate (par exemple dans 1'Intermezzo, op. $118, \mathrm{n}^{\circ} 1$ ). La musique instrumentale de tradition sérieuse comporte deux exemples, le deuxième mouvement de la Sonate pour piano $\mathrm{n}^{\circ} 3$ en fa mineur, op. 5 , et le second mouvement du Quintette à cordes $\mathrm{n}^{\circ} 1$ en fa majeur, op. 88. Dans les deux cas, la modulation au ton final s'effectue à la toute fin du mouvement, dans une section postcadentielle.

Le mouvement de la Sonate pour piano $n^{\circ} 3$ est presque entièrement dominé par le ton de la bémol, et seule la coda greffée sur la fin de la pièce module au ton de ré bémol et conclut dans cette tonalité. Bozarth affirme que cette œuvre est basée sur un programme, et suggère que l'irrégularité de l'organisation tonale

${ }^{34}$ Voir les ouvrages suivants : Harald Krebs, « Alternatives to Monotonality in Early Nineteenth-Century Music », Journal of Music Theory 25 (1981) :1-16; idem, « The Background Level in Some Tonally Deviating Works of Franz Schubert », In Theory Only 8, $n^{\circ} 8(1985)$ : 5-18; William Kinderman, « Directional Tonality in Chopin », dans Chopin Studies, édité par Jim Samson (Cambridge : Cambridge University Press, 1988), 59-76; et Carl Schachter, « Chopin's Fantasy op. 49 : The Two-Key Scheme », dans ibid., 221-53. 
est justifiée par des raisons extra-musicales ${ }^{35}$. Dans le Quintette $\mathbf{n}^{\circ} 1$, l'organisation tonale s'explique par l'organisation formelle. L'œuvre, dans son ensemble, comporte trois mouvements seulement, mais le deuxième représente en fait la combinaison de deux morceaux, un mouvement lent (en do dièse mineur) et un scherzo (en la majeur) ${ }^{36}$. Comme le montre Mahrt, la tonalité progressive résulte du fait que la pièce débute dans le ton du mouvement lent, mais se termine dans le ton du scherzo ${ }^{37}$. Les deux exemples de Brahms s'analysent mieux comme des cas particuliers de monotonalité modifiée que comme de purs exemples de tonalité progressive.

\section{CONCLUSION}

L'étude des structures à retour fait ressortir les modifications de l'utilisation du retour et de l'organisation cadentielle chez Brahms par rapport aux classiques. L'organisation cadentielle des structures à retour témoigne de l'élargissement des ressources tonales classiques, qu'il s'agisse de forme à grande ou petite échelle : d'une part, à travers l'élargissement de l'espace tonal au sein de la monotonalité, visible dans les cadences sur des degrés inhabituels, ou l'utilisation de l'exposition à trois tonalités; et d'autre part, par l'utilisation occasionnelle du complexe de double tonique dans le thème subordonné. Ainsi, Brahms ne se contente pas de modifier l'organisation interne des structures à retour (en modifiant l'organisation du retour et en développant de nouveaux patrons structurels), mais il les exploite de manière à enrichir et renouveler les principes formels de la sonate. L'usage abondant des structures à retour dans ces deux perspectives prouve l'excellente capacité d'adaptation de ce type formel aux nouvelles exigences stylistiques du romantisme.

\section{Résumé}

La difficulté de situer Brahms dans le camp des conservateurs ou des progressistes résulte probablement du caractère protéiforme de sa musique. Cette flexibilité caractérise également les structures à retour, qui sont les formes thématiques les plus abondantes dans la musique instrumentale de Brahms. Ces formes sont fondées sur le retour d'une idée initiale après un matériau intermédiaire, comme dans la période ou la forme ternaire. L'analyse des deux paramètres fondamentaux de ces structures, le retour et la cadence, montre que leur faveur peut s'expliquer par une bonne adaptation aux nouvelles exigences stylistiques du romantisme, comme le rejet de conventions trop prévisibles du discours, et l'élargissement de l'espace tonal.

35 George S. Bozarth, « Brahms's "Lieder ohne Worte" : The "Poetic" Andantes of the Piano Sonatas », dans Brahms Studies : Analytical and Historical Perspectives, 362.

36 Voir Sisman, « Brahms's Slow Movements », 83, et William Mahrt, « Brahms's Distortions of Classic Conventions ", dans Convention in Eighteenth- and Nineteenth-Century Music : Essays in Honor of Leonard G. Ratner, édité par Wye J. Allanbrook, Janet M. Levy et William P. Mahrt (Stuyvesant : Pendragon Press, 1992), 75-112, 96-98.

${ }^{37}$ Mahrt, « Brahms's Distortions of Classic Conventions », dans Convention in Eighteenth-and Nineteenth-Century Music, 96. 